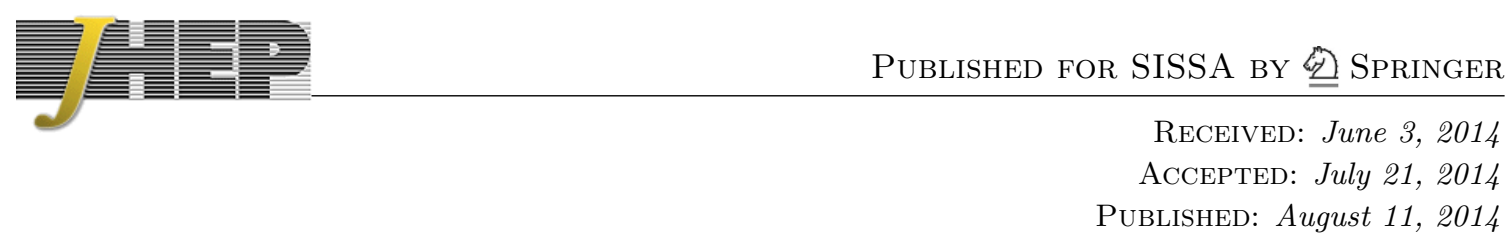

\title{
What next for the CMSSM and the NUHM: improved prospects for superpartner and dark matter detection
}

\section{Leszek Roszkowski, ${ }^{1}$ Enrico Maria Sessolo and Andrew J. Williams}

National Centre for Nuclear Research, Hoża 69, 00-681 Warsaw, Poland

E-mail: L.Roszkowski@sheffield.ac.uk,

Enrico-Maria.Sessolo@fuw.edu.pl, Andrew.Williams@fuw.edu.pl

ABSTRACT: We present an updated analysis of the CMSSM and the NUHM using the latest experimental data and numerical tools. We map out favored regions of Bayesian posterior probability in light of data from the LHC, flavor observables, the relic density and dark matter searches. We present some updated features with respect to our previous analyses: we include the effects of corrections to the light Higgs mass beyond the 2-loop order using FeynHiggs 2.10.0; we include in the likelihood the latest limits from direct searches for squarks and gluinos at ATLAS with $\sim 20 \mathrm{fb}^{-1}$; the latest constraints on the spin-independent scattering cross section of the neutralino from LUX are applied taking into account uncertainties in the nuclear form factors. We find that in the CMSSM the posterior distribution now tends to favor smaller values of $M_{\mathrm{SUSY}}$ than in the previous analyses. As a consequence, the statistical weight of the $A$-resonance region increases to about $30 \%$ of the total probability, with interesting new prospects for the $14 \mathrm{TeV}$ run at the LHC. The most favored region, on the other hand, still features multi-TeV squarks and gluinos, and $\sim 1 \mathrm{TeV}$ higgsino dark matter whose detection prospects by current and one-tonne detectors look very promising. The same region is predominant in the NUHM, although the $A$-resonance region is also present there as well as a new solution, of neutralino-stau coannihilation through the channel $\tilde{\tau} \tilde{\tau} \rightarrow h h$ at very large $\mu$. We derive the expected sensitivity of the future CTA experiment to $\sim 1 \mathrm{TeV}$ higgsino dark matter for both models and show that the prospects for probing both models are realistically good. We comment on the complementarity of this search to planned direct detection one-tonne experiments.

KEYWORDS: Supersymmetry Phenomenology

ARXIV EPRINT: 1405.4289

\footnotetext{
${ }^{1}$ On leave of absence from the University of Sheffield, U.K.
} 


\section{Contents}

1 Introduction 1

2 Scanning methodology and experimental constraints 3

3 Results in the CMSSM $\quad 8$

3.1 Posterior distributions and prospects for collider searches 8

$\begin{array}{lll}3.2 & \text { Prospects for dark matter detection } & 15\end{array}$

4 Results in the NUHM 17

$\begin{array}{lll}4.1 & \text { Posterior distributions and prospects for collider searches } & 17\end{array}$

4.2 Prospects for dark matter detection 23

5 Summary and conclusions $\quad 25$

\section{Introduction}

The recent discovery of a Higgs boson at the LHC [1,2] raised widespread excitement in the particle physics community and spurred a lot of activity to interpret the new discovery in the context of the Standard Model (SM) and models of new physics. In particular, the mass of the newly discovered particle, $m_{h} \simeq 126 \mathrm{GeV}$, is well within (albeit on the upper side) the predictions of low-scale supersymmetry (SUSY). In fact, since in SUSY the quartic coupling of the scalar potential is related to the gauge couplings of the electroweak (EW) sector, the lightest Higgs mass can deviate from the masses of the $W$ and $Z$ bosons only through radiative corrections so that its value is effectively bounded to be less than about $135 \mathrm{GeV}$.

The implications of the Higgs discovery for the parameter space of the popular Constrained Minimal Supersymmetric Standard Model (CMSSM) [3] and Non-Universal Higgs Model (NUHM), have been intensely investigated (see, e.g., [4-18] for some of the papers that followed the Higgs discovery). Many of those studies, including our own ones, explored statistical combinations of the constraints from the Higgs measurements at the LHC with other pieces of experimental information: the measurement of the dark matter relic density [19]; a number of EW precision observables; measurements of rare-decay branching ratios like $\mathrm{BR}\left(\overline{\mathrm{B}} \rightarrow \mathrm{X}_{\mathrm{s}} \gamma\right)[20], \mathrm{BR}\left(\mathrm{B}_{\mathrm{u}} \rightarrow \tau \nu\right)$ [21], or the recent measurement of a SM-like $\mathrm{BR}\left(\mathrm{B}_{\mathrm{s}} \rightarrow \mu^{+} \mu^{-}\right)$at the LHC [22, 23]; the anomalous magnetic moment of the muon $[24,25]$, etc. Additional constraints came from direct SUSY searches at the LHC. For instance, in our previous CMSSM and NUHM analyses $[11,16]$ the likelihood function included limits from the CMS razor $4.4 \mathrm{fb}^{-1}$ analysis at $7 \mathrm{TeV}[26]$ and the CMS $\alpha_{T} 11.7 \mathrm{fb}^{-1}$ analysis at $8 \mathrm{TeV}$ [27], which were obtained by simulating the SUSY signal and detector 
response and comparing them to the observed and background yields in different channels given by the experimental collaboration.

It is important to point out that, besides the Higgs boson mass measurement and LHC direct bounds, the constraint showing by far the strongest impact on the parameter space of the Minimal Supersymmetric SM (MSSM) is the relic density. It is measured very precisely and its value tends to be too large in broad regions of the parameter space. The mechanisms for reducing the relic abundance are quite general and insensitive to the particular pattern of scalar mass unification with the exception, obviously, of solutions requiring the presence of light sleptons, which in the CMSSM/NUHM are excluded by direct LHC limits on the squarks. In other words, while featuring a limited number of free parameters that make them more predictive than general phenomenological parametrizations defined at the scale of the lightest SUSY partners, the CMSSM and the NUHM produce solutions to the relic density that are present and play an important role in more general models. From this point of view, the CMSSM ansatz and its most immediate extension, the NUHM, are very useful frameworks to investigate the predictions for dark matter in constrained frameworks within the MSSM with gaugino unification at the GUT scale.

The picture that emerged particularly in the analyses $[15,16]$ for the CMSSM and [14, 16] for the NUHM, is that the relatively large value of the Higgs mass, the SM-like nature of its couplings to the other SM particles, the measured values of the flavor physics observables in great agreement with the SM, and the non-observation of SUSY particles below $1-1.5 \mathrm{TeV}$ at the LHC, can all be easily accommodated in an extended region of the parameter space characterized by squarks and gluinos in the multi- $\mathrm{TeV}$ regime and the heavy Higgs sector effectively decoupled. In this region, the relic density can naturally assume the value measured by PLANCK, as the lightest SUSY particle (LSP) is an almost pure higgsino neutralino with a mass $m_{\chi} \simeq 1 \mathrm{TeV} .{ }^{1}$ The existence of the $\sim 1 \mathrm{TeV}$ higgsino solution for DM in the MSSM has been long known [28, 29] but in the framework of unified SUSY was first pointed out in a pre-LHC study of the NUHM [30].

Interestingly, parameter space regions with sparticles in the multi-TeV regime and $\sim 1 \mathrm{TeV}$ higgsino dark matter in agreement with the value of the relic density were shown to be very favored also in scans of the phenomenological MSSM [31], in the Next-toMinimal Supersymmetric SM [32], and in a variety of models with non-universal boundary conditions at the GUT scale [33]. Incidentally, it was shown in [33] that for some of these models focus point-like mechanisms significantly increase the naturalness of the $\sim 1 \mathrm{TeV}$ higgsino region with respect to the CMSSM, without affecting dark matter properties and prospects for detection. As a matter of fact, the best prospects for detection of the $\sim 1 \mathrm{TeV}$ higgsino region indeed come from dark matter direct detection experiments, particularly at 1-tonne detectors, as the spin-independent neutralino-proton cross section, $\sigma_{p}^{\mathrm{SI}}$, is well within the projected sensitivities of the currently running and future 1-tonne experiments.

\footnotetext{
${ }^{1}$ While other regions of the parameter space, characterized by bino dark matter and such that the relic abundance is saturated through mechanisms of $A$-resonance or stau co-annihilation, are consistent with the constraints at the 1 or $2 \sigma$ level, the $\sim 1 \mathrm{TeV}$ higgsino region shows by far the best agreement with all of them with the exception of $\delta(g-2)_{\mu}$, which favors low $M_{\text {SUSY }}$ below ATLAS/CMS bounds in unified models.
} 
Also very interestingly, recent studies of the sensitivity of the Cherenkov Telescope Array (CTA) [34] show the largest projected reach in the region characterized by dark matter mass and annihilation cross section typical of the $\sim 1 \mathrm{TeV}$ higgsino region $[35,36]$, thus opening up the enticing possibility of complementary detection for these scenarios.

On the other hand, the statistical analyses of refs. [9-16] were based on the calculation of the Higgs mass performed at two loops, implemented in the most popular SUSY spectrum calculators [37-39] or in earlier versions of FeynHiggs [40-43]. Significant effort in the direction of improving the theoretical precision of the Higgs mass calculation in the MSSM [44-48] has recently prompted some groups to update their previous analyses [4951]. Reference [51] in particular shows the first global statistical analysis to incorporate these recent developments in a frequentist approach.

In this paper, we update the global Bayesian analyses of the CMSSM and the NUHM previously produced by our group, by including the following new elements:

- We calculate the Higgs mass with FeynHiggs 2.10.0 [47], which incorporates results beyond two loops with a resummation of leading and sub-leading logarithms in the top/stop sector.

- We update the likelihood map for direct SUSY searches with the most constraining limits from ATLAS with $20 \mathrm{fb}^{-1}$ at $8 \mathrm{TeV}$.

- We include the recent constraints from the LUX experiment [52] in the likelihood function.

- We add an analysis of the prospect for CTA to independently explore the favored regions.

We will focus in particular on the properties of the regions that present the highest posterior probability, favored by the value of the dark matter relic density. We will show that the bulk of the posterior still lies on the $\sim 1 \mathrm{TeV}$ higgsino region, but in the CMSSM its statistical significance is not as overwhelmingly predominant as previously shown. We will touch on the prospects for detection at the LHC and in future colliders, but will focus particularly on dark matter searches like the above-mentioned 1-tonne detectors and the CTA.

The paper is organized as follows. In section 2 we describe the statistical setup, the constraints included in the likelihood function, the parameter prior ranges and distributions, and the numerical tools used in our scans. In section 3 we present the results of the Bayesian analysis of the CMSSM, including prospects at collider and dark matter experiments. In section 4 we present equivalent results for the NUHM. We give our summary and conclusions in section 5 .

\section{Scanning methodology and experimental constraints}

Our goal is to determine the regions in the parameter space of the CMSSM and the NUHM that are favored by all of the experimental data available. We follow a Bayesian approach outlined in $[11,16,53,54]$ and map out the $68 \%$ and $95 \%$ credible regions in 
two-dimensional (2D) projections of the marginalized posterior probability density function (pdf) and/or the one-dimensional (1D) marginalized pdf of some interesting parameters. The posterior pdf (or, simply, the posterior), $p(m \mid d)$, is given by Bayes' Theorem,

$$
p(m \mid d)=\frac{p(d \mid m) \pi(m)}{p(d)}
$$

where $m$ is the set of model parameters, $\pi(m)$ is prior probability distribution of the parameters $m, p(d \mid m) \equiv \mathcal{L}(m)$ is the probability of obtaining the experimental data $d$ given the model parameters, known as the likelihood function, and $p(d)$ is a normalization factor called the evidence, which is required for the comparison of different models. In this framework the likelihood function encodes all of our information from experimental constraints and their associated uncertainties.

We can consider marginalized posterior distributions of subsets of the parameters $m$ by integrating over the remaining parameters. The posterior distribution of a subset of parameters $\psi_{1 \ldots r}$ from the full set of parameters $m_{1 \ldots N}$ is then given by

$$
p\left(\psi_{1 \ldots r} \mid d\right)=\int p(m \mid d) d^{N-r} m .
$$

This leads to a natural prescription for dealing with nuisance parameters, since these can be included as parameters of the model with suitable prior probability distributions and then marginalized over to obtain posterior distributions of the parameters of interest.

We construct the likelihood function from the experimental data. We account for positive measurements with a gaussian likelihood function and combine experimental and theoretical errors in quadrature. A summary of the experimental constraints used in this analysis is given in table 1 . In addition, we adopt a specific procedure for incorporating the constraints from Higgs boson searches at the LHC, direct searches for SUSY, and the constraints from dark matter direct detection at LUX.

The discovery of a SM-like Higgs boson with $m_{h} \simeq 126 \mathrm{GeV}$ presents a challenging constraint. The experimental collaborations have published the Higgs signal rates in several channels and we make use of this information by interfacing with the public code HiggsSignals v1.0.0 [55]. We supply HiggsSignals with the Higgs boson production cross section and branching ratios calculated by FeynHiggs [40-43] and use the calculated $\chi^{2}$ result in the likelihood function. An accurate prediction for the lightest Higgs boson is important due to the small width of the observed experimental signal. Recently [47] FeynHiggs v2.10.0 has incorporated results beyond two loops with a resummation of leading and sub-leading logarithms in the top/stop sector. We include these in our scan. We fix the uncertainty in the mass of the lightest Higgs to $2 \mathrm{GeV}$, as a conservative estimate of the remaining sources of theory error. As well as the observed signal at $\sim 126 \mathrm{GeV}$, Higgs searches in other mass ranges may constrain the heavy Higgs bosons. We use HiggsBounds v4.1.0 [56-58] to reject points excluded at 95\% C.L. by those searches.

The contribution to the likelihood arising from the results of the LUX experiment [52] is derived as was explained in [33], i.e., by applying the procedure developed in ref. [69] for the likelihood of XENON100 [70] to the data from LUX. We assume that the number 


\begin{tabular}{|c|c|c|c|c|}
\hline Constraint & Mean & Exp. Error & Th. Error & Ref. \\
\hline Higgs sector & See text. & See text. & See text. & {$[55-58]$} \\
\hline Direct SUSY searches & See text. & See text. & See text. & {$[59-67]$} \\
\hline$\sigma_{p}^{\text {SI }}$ & See text. & See text. & See text. & {$[52]$} \\
\hline$\Omega_{\chi} h^{2}$ & 0.1199 & 0.0027 & $10 \%$ & {$[19]$} \\
\hline $\sin ^{2} \theta_{\text {eff }}$ & 0.23155 & 0.00015 & 0.00015 & {$[68]$} \\
\hline$\delta(g-2)_{\mu} \times 10^{10}$ & 28.7 & 8.0 & 1.0 & {$[24,25]$} \\
\hline $\mathrm{BR}\left(\overline{\mathrm{B}} \rightarrow \mathrm{X}_{\mathrm{s}} \gamma\right) \times 10^{4}$ & 3.43 & 0.22 & 0.21 & {$[20]$} \\
\hline $\mathrm{BR}\left(\mathrm{B}_{\mathrm{u}} \rightarrow \tau \nu\right) \times 10^{4}$ & 0.72 & 0.27 & 0.38 & {$[21]$} \\
\hline$\Delta M_{B_{s}}$ & $17.719 \mathrm{ps}^{-1}$ & $0.043 \mathrm{ps}^{-1}$ & $2.400 \mathrm{ps}^{-1}$ & {$[68]$} \\
\hline$M_{W}$ & $80.385 \mathrm{GeV}$ & $0.015 \mathrm{GeV}$ & $0.015 \mathrm{GeV}$ & {$[68]$} \\
\hline $\mathrm{BR}\left(\mathrm{B}_{\mathrm{s}} \rightarrow \mu^{+} \mu^{-}\right) \times 10^{9}$ & 2.9 & 0.7 & $10 \%$ & {$[22,23]$} \\
\hline
\end{tabular}

Table 1. The experimental constraints used in this study.

of observed events follows a Poisson distribution centered on the predicted signal plus background. A likelihood map in the $\left(m_{\chi}, \sigma_{p}^{\mathrm{SI}}\right)$ plane is generated by simulating signal events in micrOMEGAs [71] and marginalizing over the uncertainty in the expected number of background events. In figure 1(a) we plot the $68.3 \%, 90 \%$, and $99.7 \%$ C.L. exclusion bounds obtained with our procedure. The dashed black line gives the official 90\% C.L. exclusion bound. In our scans, we also account for uncertainties in the predicted elastic scattering cross section $[72,73]$ by including the nuclear form factors $\sigma_{s}$ and $\Sigma_{\pi N}$ as nuisance parameters.

We finally account for the direct SUSY searches at the LHC by updating the method developed in $[11,16]$. We generate a grid in the $\left(m_{0}, m_{1 / 2}\right)$ plane at $50-\mathrm{GeV}$ intervals. At each point we generate squark- and gluino-production events using Madgraph [74] and produce the parton shower in pythia [75]. The cross sections are calculated using nll fast [76-80] to include the next-to-leading order and next-to-leading log contributions. We evaluate the expected number of events in a given signal region for the searches considered using CheckMATE [59-67]. CheckMATE includes a number of validated SUSY searches and includes an advanced tuning of the fast detector simulation. We calculate a likelihood for each search from the product of Poisson distributions for each signal region. We account for the uncertainties in the background rate by marginalizing over the background rate with a gaussian distribution. When calculating the likelihood, we consider the two searches that give the strongest limits in the CMSSM: a 0 lepton 2-6 jets ATLAS search [81] and a 0-1 lepton $3 b$-jets ATLAS search [82]. We scale the total squark and gluino production rate by a small constant factor to match the limit achieved by the experimental analyses in order to account for the remaining differences in efficiencies due to the fast detector simulation. To combine the results of the two ATLAS searches we evaluate at each point which of the two searches has the largest expected exclusion and then use that search to 


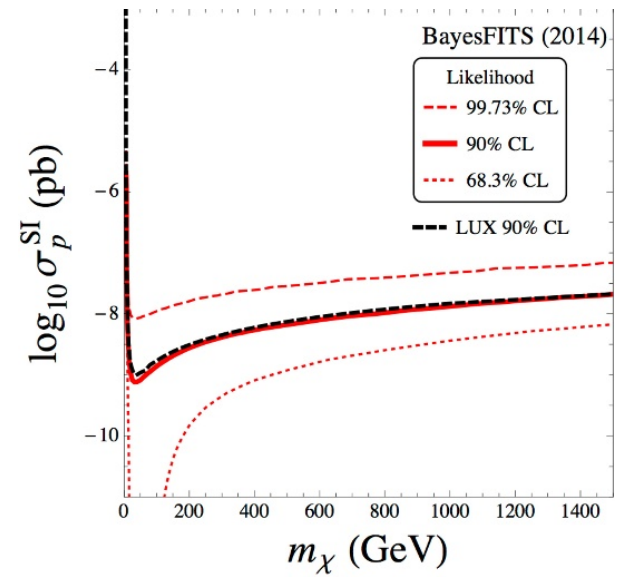

(a)

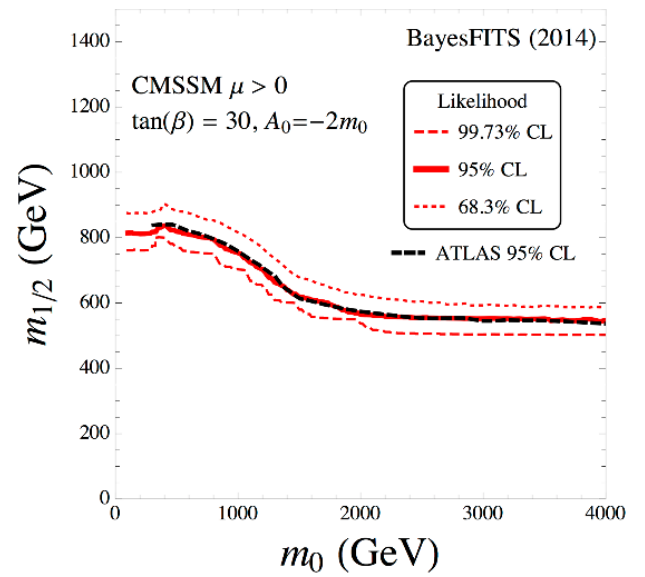

(b)

Figure 1. (a) The $68.3 \%$ C.L. (red dotted line), 90\% C.L. (red solid line) and 99.7\% C.L. (red dashed line) exclusion bounds given by our likelihood map for dark matter direct detection experiments, compared with the 90\% C.L. limit published by LUX [52] (black dashed line). (b) The $68.3 \%$ C.L. (red dotted line), 95\% C.L. (red solid line) and 99.7\% C.L. (red dashed line) exclusion bounds for our combination of the ATLAS searches compared to the original 95\% C.L. contour from ATLAS (black dashed line).

calculate the likelihood for that point. The combination of the two searches compared to the official 95\% C.L. line from ATLAS (where we have combined the lines from the two different searches) is shown in figure 1(b). The agreement is very good across the entire range of $m_{0}$ and $m_{1 / 2}$.

In this study, we also estimate the sensitivity of the Cherenkov Telescope Array (CTA) [34] for the favored parameter space of the CMSSM and the NUHM. The results will be shown in section 3.2 and section 4.2 , respectively.

The CTA project will build the next generation air Cherenkov telescope observatory. For dark matter masses greater than $\sim 100 \mathrm{GeV}$ CTA is expected to significantly exceed current experimental limits for dark matter annihilations such as those from HESS [83] and Fermi-LAT [84]. CTA may even probe cross sections below the canonical thermal relic value for some final states [36].

Reference [36] estimated the future sensitivity of CTA to dark-matter annihilation in the Galactic Center (GC) for the final states $b \bar{b}, \mu^{+} \mu^{-}$and $\tau^{+} \tau^{-}$assuming 500 hours of observation time. In order to directly apply their limits to more generic neutralino annihilations, whose final states also include gauge bosons, $Z Z$ and $W^{+} W^{-}$, we infer from [36] the $95 \%$ C.L. limit on the expected flux of signal photons per $J$-factor, $N_{\gamma, 95}$, by convolving the photon flux with the effective area given in [85] in a single energy bin between $30 \mathrm{GeV}$ and the dark matter mass:

$$
N_{\gamma, 95}=t_{\mathrm{obs}} \frac{\langle\sigma v\rangle_{95}^{\left(b \bar{b}, \mu^{+} \mu^{-}, \tau^{+} \tau^{-}\right)}}{8 \pi m_{\chi}^{2}} N_{\gamma, \text { obs }} J_{\text {fact }},
$$

where $t_{\mathrm{obs}}$ is the observation time, $\langle\sigma v\rangle_{95}$ is the projected $95 \%$ C.L. limit for each final 


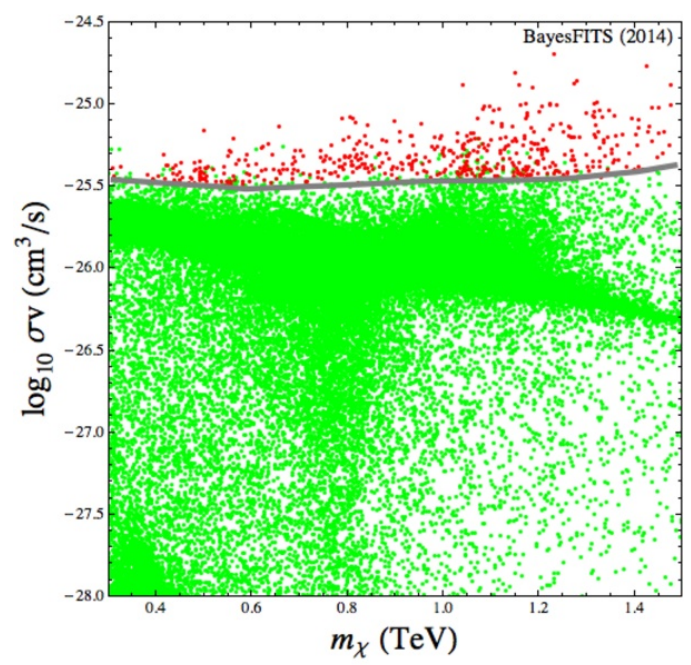

Figure 2. Our indicative 95\% C.L. projected sensitivity in the $\left(m_{\chi}, \sigma v\right)$ plane of the MSSM for $500 \mathrm{~h}$ of observation at CTA is shown as a gray solid line. The red (dark) points are excluded at the $95 \%$ C.L. through direct comparison with $N_{\gamma, 95}$, which we derived from the projected bounds given in [36] for different annihilation final states. The gray line marks the maximum cross section bound, which for each point depends on the individual final states.

state, and $N_{\gamma, \text { obs }}$ is given by

$$
N_{\gamma, \mathrm{obs}}=\int_{30 \mathrm{GeV}}^{m_{\chi}} \frac{d N_{\gamma}(E)}{d E} A_{\mathrm{eff}}(E) d E,
$$

where $A_{\text {eff }}$ is the effective area, $d N_{\gamma}(E) / d E$ is the energy spectrum per annihilation and for simplicity we have neglected the effect of finite energy resolution. For validation, one must make sure that the $N_{\gamma, 95}$ obtained from the different final-state bounds of [36] are all in very good agreement.

Once $N_{\gamma, 95}$ is derived, the photon flux is calculated point by point in our scans using micrOMEGAs. In figure 2 we show the approximate $95 \%$ C.L. limit in the plane of the annihilation cross section times velocity at zero momentum, $\sigma v \equiv \lim _{p \rightarrow 0}\langle\sigma v\rangle$, versus the neutralino mass, $m_{\chi}$. The limit is extracted by testing each point against the expected $N_{\gamma, 95}$, and showing them in red if they are excluded. The large statistical sample shown in figure 2 includes the scans performed for this study and those used in [31].

Note that the limit is derived for the photon flux, so that there is no corresponding clear-cut limit in $\sigma v$. Nevertheless, we show with a gray solid line the maximum extent that can be probed with this method, and we will apply the obtained limit to the figures in section 3.2 and section 4.2 .

The scans in this study are performed with the BayesFITS package $[11,16,31,86]$, which interfaces several publicly available tools to direct the scanning procedure and calculate physical observables. The sampling is performed by MultiNest [87] with 4000 and 10000 live points for the CMSSM and NUHM respectively. The evidence tolerance is set to 0.5 and the sampling efficiency to 0.8 for all the scans. We use SoftSusy v.3.3.9 [37] to calculate the mass spectrum. As was explained above, this is passed via the SUSY 


\begin{tabular}{|c|c|c|c|}
\hline Parameter & Description & Range & Distribution \\
\hline$m_{0}$ & Universal scalar mass & $0.1,20$ & Log \\
\hline$m_{1 / 2}$ & Universal gaugino mass & $0.1,10$ & Log \\
\hline$A_{0}$ & Universal trilinear coupling & $-20,20$ & Linear \\
\hline $\tan \beta$ & Ratio of the Higgs vevs & 3,62 & Linear \\
\hline $\operatorname{sgn} \mu$ & Sign of the Higgs/higgsino mass parameter & +1 or -1 & \\
\hline$m_{H_{d}}^{2} /{\sqrt{\left|m_{H_{d}}^{2}\right|^{(*)}}}^{2}$ & Signed GUT-scale soft mass of $H_{d}$ & $-20,20$ & Linear \\
\hline$m_{H_{u}}^{2}{\sqrt{\left|m_{H_{u}}^{2}\right|^{(*)}}}_{\text {Nuisance parameter }}^{\text {Signed GUT-scale soft mass of } H_{u}}$ & $-10,10$ & Linear \\
\hline$M_{t}$ & Description & Central value & Distribution \\
\hline$m_{b}\left(m_{b}\right)^{\overline{M S}}$ & Top quark pole mass & $173.34 \pm 0.76 \mathrm{GeV}[90]$ & Gaussian \\
\hline$\alpha_{s}\left(M_{Z}\right)^{\overline{M S}}$ & Bottom quark mass & $4.18 \pm 0.03 \mathrm{GeV}[68]$ & Gaussian \\
\hline $1 / \alpha_{\mathrm{em}}\left(M_{Z}\right)^{\overline{M S}}$ & Strong coupling & $0.1185 \pm 0.0006[68]$ & Gaussian \\
\hline$\Sigma_{\pi N}$ & Reciprocal of electromagnetic coupling & $127.944 \pm 0.014[68]$ & Gaussian \\
\hline$\sigma_{s}$ & Nucleon sigma term & $34 \pm 2 \mathrm{MeV}[71]$ & Gaussian \\
\hline & Strange sigma commutator & $42 \pm 5 \mathrm{MeV}[71]$ & Gaussian \\
\hline
\end{tabular}

(*) These quantities are independently scanned in the NUHM analysis.

Table 2. Prior distributions of the CMSSM and nuisance parameters used in the scans. All dimensionful parameters are given in $\mathrm{TeV}$ unless indicated otherwise.

LesHouches Accord format to FeynHiggs v.2.10.0 to calculate the higher-order corrections to the Higgs mass. FeynHiggs is interfaced with HiggsSignals and HiggsBounds to evaluate the constraints on the Higgs sector. SuperISO v.3.3 [88] is used to calculate $\mathrm{BR}\left(\overline{\mathrm{B}} \rightarrow \mathrm{X}_{\mathrm{s}} \gamma\right), \mathrm{BR}\left(\mathrm{B}_{\mathrm{s}} \rightarrow \mu^{+} \mu^{-}\right),{ }^{2} \mathrm{BR}\left(\mathrm{B}_{\mathrm{u}} \rightarrow \tau \nu\right)$, and $\delta(g-2)_{\mu}$. The observables $M_{W}$, $\sin ^{2} \theta_{\text {eff }}, \Delta M_{B_{s}}$ are calculated using FeynHiggs. The dark matter observables $\Omega_{\chi} h^{2}, \sigma_{p}^{\mathrm{SI}}$, and $\sigma v$ are computed using micrOMEGAs v.3.5.5 [71].

The prior distributions of the model and nuisance parameters for the CMSSM are given in table 2. Additionally the parameters scanned in the NUHM are indicated with an asterisk. The sign of $\mu$ is fixed for each scan. Note that for $\tan \beta<3$ it becomes very difficult to obtain at the same time EWSB, the correct value of the relic density, or the correct Higgs mass, as the parameters $m_{H_{u}}, m_{H_{d}}$, $\tan \beta$, and the one-loop tadpole corrections must be fine-tuned very precisely. Note also that for the scans with $\mu<0$ we do not include $\delta(g-2)_{\mu}$ in the likelihood as it is known to be poorly fitted. We use logarithmic priors for the universal mass parameters $m_{0}$ and $m_{1 / 2}$ to reduce volume effects.

\section{Results in the CMSSM}

\subsection{Posterior distributions and prospects for collider searches}

In figure 3(a) we show the $68 \%$ and $95 \%$ credible regions of the marginalized $2 \mathrm{D}$ pdf in the $\left(m_{0}, m_{1 / 2}\right)$ plane of the CMSSM, for $\mu>0$. The gray dashed contours mark the previous

\footnotetext{
${ }^{2}$ We use the non time-averaged output of SuperISo, as explained in [31]. It is numerically closer to the time-averaged SM prediction of ref. [89] than the code's own time-averaged calculation.
} 


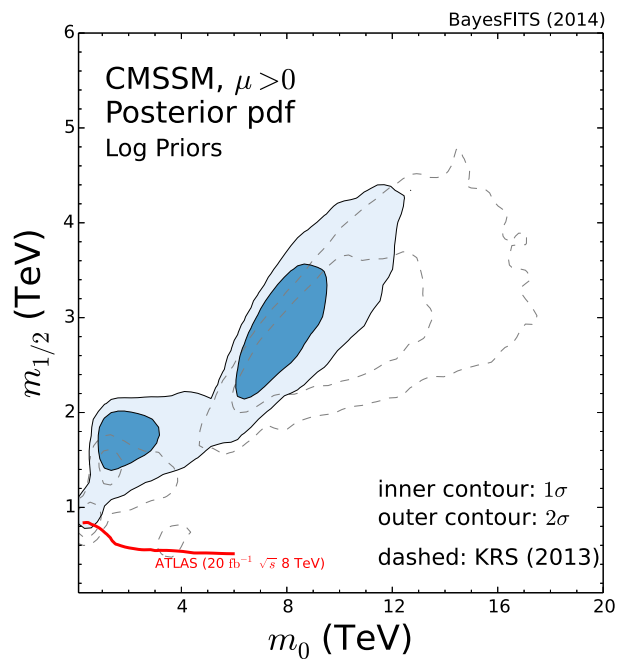

(a)

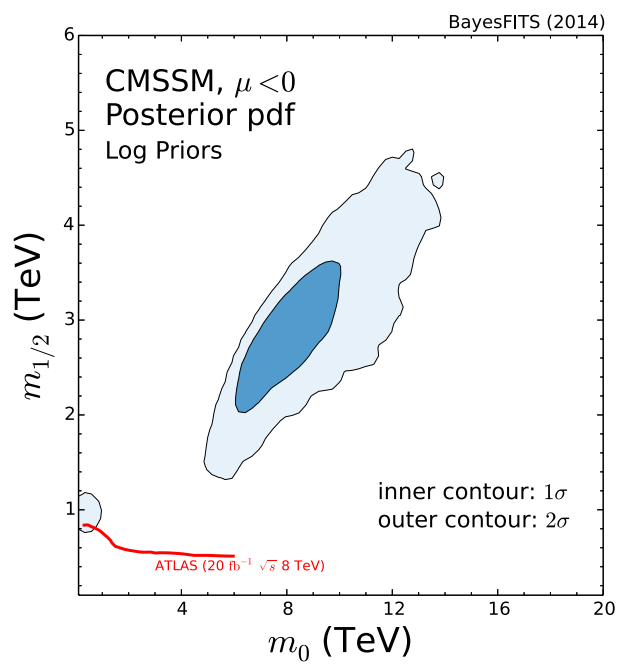

(c)

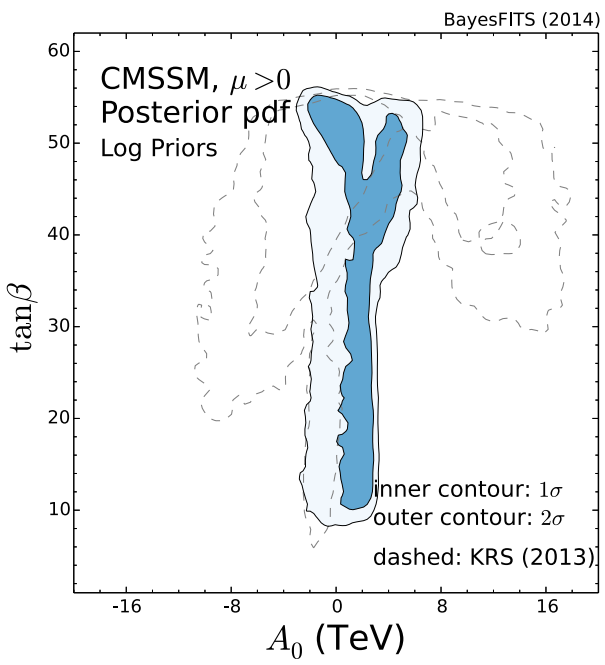

(b)

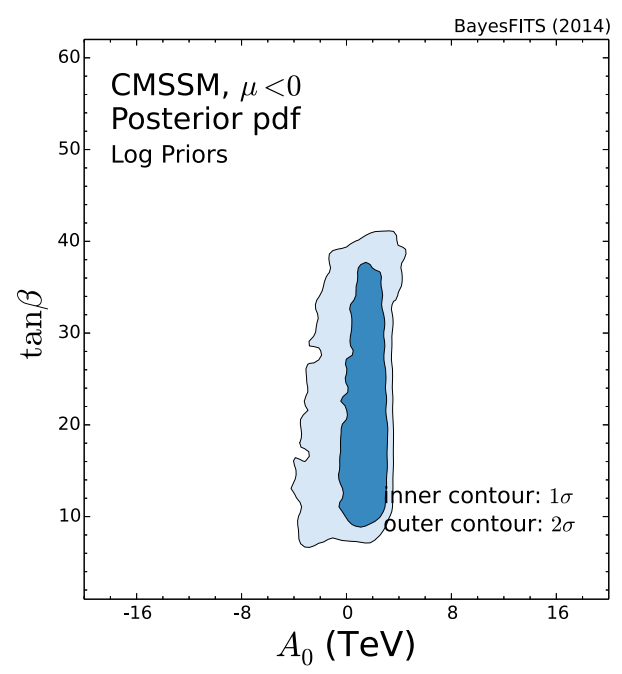

(d)

Figure 3. Marginalized 2D posterior distribution for the CMSSM in (a) the $\left(m_{0}, m_{1 / 2}\right)$ plane for $\mu>0$, (b) the $\left(A_{0}, \tan \beta\right)$ plane for $\mu>0$, (c) the $\left(m_{0}, m_{1 / 2}\right)$ plane for $\mu<0$, and (d) the $\left(A_{0}\right.$, $\tan \beta$ ) plane for $\mu<0$. The $68 \%$ credible regions are shown in dark blue and the $95 \%$ credible regions in light blue. For comparison we show the $68 \%$ and $95 \%$ credible regions of [16] (KRS (2013) hereafter) encapsulated by thin gray dashed lines. The ATLAS 95\% C.L. exclusion line is shown as a red solid line for reference. 
$68 \%$ and $95 \%$ regions obtained in [16], which we present for comparison to highlight the impact of the new more precise calculation of $m_{h}$ and the new improved constraints.

As has been long standing practice, in the CMSSM the modes of the posterior pdf are identified according to the respective mechanisms to satisfy the relic density constraint. The little, round, $95 \%$ credibility region just above the ATLAS line at low $m_{0}$ is the staucoannihilation region [91]; the large region immediately above it, for $m_{0} \lesssim 5 \mathrm{TeV}$ and $m_{1 / 2} \gtrsim 1.2 \mathrm{TeV}$ is the $A$-resonance region [92]; the remaining mode for $m_{0}>5 \mathrm{TeV}$ and $m_{1 / 2} \gtrsim 1.5 \mathrm{TeV}$ is the above-mentioned $\sim 1 \mathrm{TeV}$ higgsino region.

The different shape of the present pdf relative to the one given in [16] is due to the new higher-order determination of the Higgs mass. Over the whole parameter space, given equivalent $M_{\mathrm{SUSY}}=\left(m_{\tilde{t}_{1}} m_{\tilde{t}_{2}}\right)^{1 / 2}$, the value of the Higgs mass has increased by about $2 \mathrm{GeV}$. In practice, this means that in the $\sim 1 \mathrm{TeV}$ higgsino region, where the Higgs mass constraint is always more easily satisfied thanks to the large scalar masses, the favored $m_{0}$ values are now limited to less than $12 \mathrm{TeV}$, above which the Higgs mass becomes too heavy. In the $A$-resonance and stau-coannihilation regions, the Higgs mass value has increased from an average $122-123 \mathrm{GeV}$ to values closer to $126 \mathrm{GeV}$, thus improving the $\chi^{2}$. As a consequence, the statistical weight of the $A$-resonance region has now much increased, with new, improved prospects for collider phenomenology, as we shall see later.

In spite of higher mass values for the Higgs boson, the stau-coannihilation region is now becoming disfavored by increasing tension with direct SUSY limits at the LHC, and it will be most likely probed in its entirety in the $14 \mathrm{TeV}$ run. Note also that, with respect to [16], the focus point region [93-95], which is shown in figure 3(a) as a $95 \%$ dashed contour just above the ATLAS line at $m_{0} \simeq 4 \mathrm{TeV}$, is now disfavored. Interestingly enough, the new estimate for the Higgs mass in the focus point region is now much closer to the experimental value than before, but the region is disfavored by the LUX results: as is well known, the neutralino there is a mixed composition of bino and higgsino with a mass of $m_{\chi} \lesssim 600 \mathrm{GeV}$. Its large $\sigma_{p}^{\mathrm{SI}}$ is now excluded by LUX at $90 \%$ C.L. We will come back to this point in section 3.2 .

In figure $3(\mathrm{~b})$ we show the credible regions of the $2 \mathrm{D}$ pdf in the $\left(A_{0}, \tan \beta\right)$ plane of the CMSSM, for $\mu>0$. The three favored modes of the posterior are not as clearly separated as in figure $3(\mathrm{a})$, or as they were in the $\left(A_{0}, \tan \beta\right)$ plane shown in [16]. One can now recognize an elongated $68 \%$ credible region for $0 \lesssim A_{0} \lesssim 2 \mathrm{TeV}$ and $8 \lesssim \tan \beta \lesssim 45$, which belongs to the $\sim 1 \mathrm{TeV}$ higgsino region and extends to slightly larger, positive $A_{0}$ values for $\tan \beta \gtrsim 45$. An elongated $95 \%$ credible area is adjacent to it to the left: it encompasses part of the $\sim 1 \mathrm{TeV}$ higgsino region, and the stau-coannihilation region. Finally, a roundish $68 \%$ and $95 \%$ credibility region at $-3 \mathrm{TeV} \lesssim A_{0} \lesssim 2 \mathrm{TeV}$ and large $\tan \beta$ is the $A$-resonance region.

The most striking difference with the results of [16] is that now the favored $A_{0}$ range is much reduced, from a maximum span of approximately $28-30 \mathrm{TeV}$, covering almost the full parameter space, to the present span of about $10 \mathrm{TeV}$, centered around zero. By comparing the solid contours in color with the previous ones in dashed lines, one can see that the two modes that were previously predominant at large $\left|A_{0}\right|$ in the $\sim 1 \mathrm{TeV}$ higgsino region are not as favored in the present scan and do not appear at $95 \%$ credibility. This is because in [16] one needed solutions with larger $M_{\text {SUSY }}$ to fit the Higgs mass measurement. When $m_{0}$ becomes very large, electroweak-symmetry breaking (EWSB) can only be obtained 


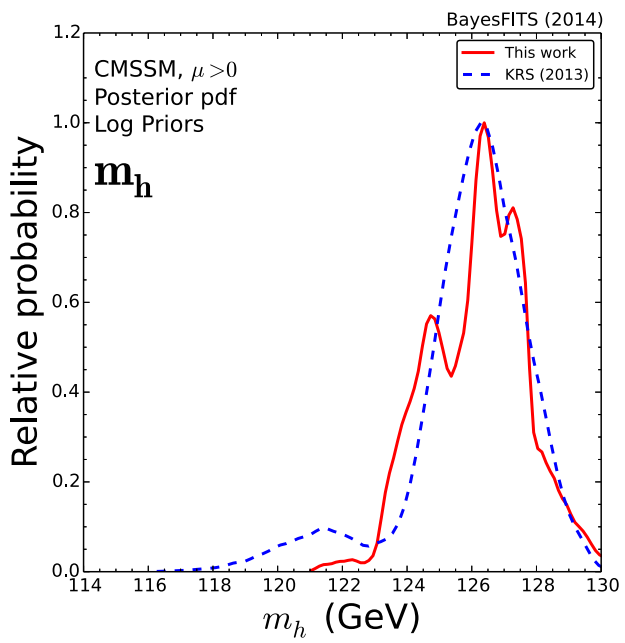

(a)

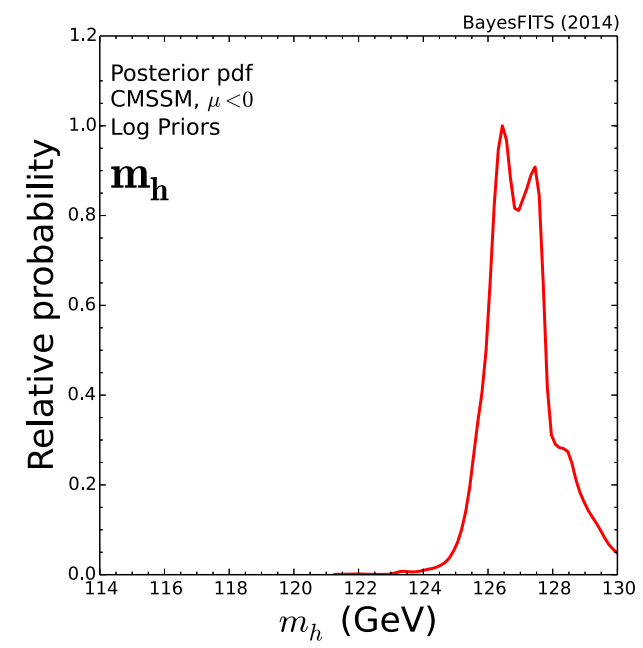

(b)

Figure 4. Marginalized 1D pdf of $m_{h}$ for the CMSSM with (a) $\mu>0$ and (b) $\mu<0$. Red solid lines is this work, blue dashed line represents the distribution obtained in KRS 2013.

with large trilinear couplings, which enhance the RGE running of $m_{H_{u}}^{2}$. However, as we said above, the correct value of the Higgs mass can now be obtained with an $M_{\text {SUSY }}$ on average smaller, even in the $\sim 1 \mathrm{TeV}$ higgsino region. Thus, the scanning program can more naturally find solutions with a reduced $\left|A_{0}\right|$.

In figure $3(\mathrm{c})$ we show the $68 \%$ and $95 \%$ credible regions of the $2 \mathrm{D}$ pdf in the $\left(m_{0}\right.$, $m_{1 / 2}$ ) plane of the CMSSM for $\mu<0$. Two of the modes of the posterior are the same as in the $\mu>0$ case, with the exception of the $A$-resonance region, which does not appear for negative $\mu$ because the conditions for EWSB are not met there. ${ }^{3}$ In figure $3(\mathrm{~d})$ we show the corresponding $2 \mathrm{D}$ pdf in the $\left(A_{0}, \tan \beta\right)$ plane.

In figure $4(\mathrm{a})$ we show in solid red the marginalized $1 \mathrm{D}$ posterior distribution for the lightest Higgs mass. We also show with a dashed blue line the distribution obtained in [16], to facilitate comparison. The posterior is in good agreement with the previous study, in which the Higgs-mass likelihood was a simple gaussian function centered about the measured CMS value, with experimental and theoretical uncertainties added in quadrature. The present likelihood function is instead determined by the sum of the $\chi^{2}$ contributions of the individual Higgs searches, encoded in the HiggsSignals program. Note that the two main peaks in the distribution, characteristic of the $A$-resonance region about $124-125 \mathrm{GeV}$,

\footnotetext{
${ }^{3}$ To be precise, the $A$-resonance region is absent not because it is disfavored by any particular constraint, but because at large $\tan \beta$ SoftSusy easily incurs negative values of the $\overline{D R}$ pseudoscalar mass squared, $m_{A}^{2}(\overline{D R})$, during the iterative procedure of RGE running. The matter was solved in earlier versions of the program by switching to the pole mass at each iteration while looking for convergence. It is debatable whether such a solution is enough to produce the correct EWSB. Thus, following the default setting in recent versions of SoftSusy, we have decided not to include the points of the $A$-resonance region for $\mu<0$. We also abstain from showing a direct comparison with the results of [16] in this case, as they were obtained with an earlier modified version of SoftSusy.
} 
and of the $\sim 1 \mathrm{TeV}$ higgsino region for larger $m_{h}$, are now much more in agreement with each other than in the previous study. This is the effect of including the higher order Higgs corrections: there are virtually no regions left in the parameter space for which constraints other than the Higgs searches push the posterior distribution toward $m_{h}<123 \mathrm{GeV}$.

The equivalent distribution for the case with $\mu<0$ is shown in figure 4 (b). In figure 5 we show the marginalized 1D posteriors for the heavy Higgs bosons and a selection of superpartner masses for $\mu>0$. Again, the dashed blue line in the figures shows the distribution for the scan of ref. [16].

In figure 5(a) we show the pdf for the mass of the lightest neutralino. The posterior presents three main modes, corresponding to the regions defined in figure 3(a). From the left to the right one can can see: the stau-coannihilation region, with $0.3 \mathrm{TeV} \lesssim m_{\chi} \lesssim$ $0.6 \mathrm{TeV}$, subtending approximately $1 \%$ of the total probability; the $A$-resonance region, $0.6 \mathrm{TeV} \lesssim m_{\chi} \lesssim 0.9 \mathrm{TeV}$, with a probability of $\sim 30 \%$; and the $\sim 1 \mathrm{TeV}$ higgsino region for $m_{\chi} \gtrsim 0.9 \mathrm{TeV}$, with $\sim 69 \%$ probability. The inclusion of higher order corrections to the Higgs mass has caused the shift of a substantial fraction of posterior probability to the $A$ resonance region. As the dashed blue line shows, the $\sim 1 \mathrm{TeV}$ higgsino region was strongly favored in [16], featuring $\sim 94 \%$ of the total probability, while the remainder was split in approximately equal parts between the other two regions. Note also that the average $m_{\chi}$ in the $\sim 1 \mathrm{TeV}$ higgsino region is slightly larger than in [16], as the PLANCK-measured value of the relic density is a little larger than the one measured by WMAP [96].

The 1D pdf for the heavy Higgs masses is shown in figure 5(b). As could be expected, the distribution features a sharp peak in the $A$-resonance region, for $m_{A} \approx 2 m_{\chi} \lesssim 2.5 \mathrm{TeV}$. The $\sim 1 \mathrm{TeV}$ higgsino region is instead characterized by a much broader range of values, extending up to $\sim 15 \mathrm{TeV}$. We will show below that a large fraction of the $A$-resonance regions is within the reach of Higgs searches at the LHC $14 \mathrm{TeV}$ run.

The 1D posterior distributions for the mass of the lightest stop, $m_{\tilde{t}_{1}}$, and that of the left-handed first and second generation squarks, generically indicated with $m_{\tilde{u}_{L}}$, are shown in figures 5(c) and 5(d), respectively. While the lightest squark masses in the figures, typical of the stau-coannihilation region, are now disfavored by the present limits from direct SUSY searches at the LHC, the increased relevance of the $A$-resonance region, which features in general lighter squarks than the $\sim 1 \mathrm{TeV}$ higgsino region, leads to a moderate optimism for squark detection in future-generation colliders. In particular, by comparing the solid red and dashed blue lines in, e.g., figures 5(c) and 5(a), one can see that in [16] there was a $\sim 30 \%$ of probability favoring $m_{\tilde{t}_{1}} \gtrsim 8 \mathrm{TeV}$ (with $m_{\chi} \gtrsim 1 \mathrm{TeV}$ ) that has now been virtually erased. On the other hand, the parameter space featuring $m_{\tilde{t}_{1}} \lesssim 4 \mathrm{TeV}$ (with $m_{\chi} \lesssim 0.8 \mathrm{TeV}$ ) is now favored by roughly the same odds.

Equivalently, one can see in figure 5(e), where we show the 1D pdf for the gluino mass $m_{\tilde{g}}$, that the increased relevance of the $A$-resonance region leads to the emergence of a new peak in probability at $m_{\tilde{g}} \simeq 3-4 \mathrm{TeV}$.

Finally, we show in figure $5(\mathrm{f})$ the $1 \mathrm{D}$ posterior for the lightest chargino mass, $m_{\chi_{1}^{ \pm}}$. The bulk of the probability is subtended by the $\sim 1 \mathrm{TeV}$ higgsino region, in which the lightest chargino is also higgsino-like and almost degenerate with the neutralino, in the 


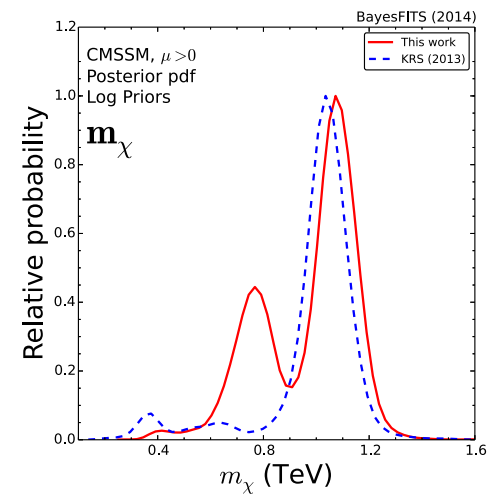

(a)

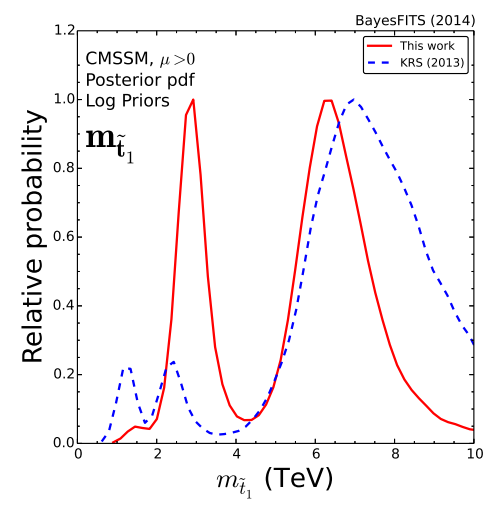

(c)

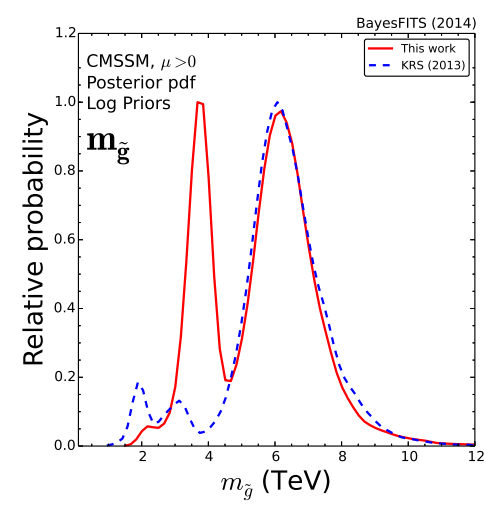

(e)

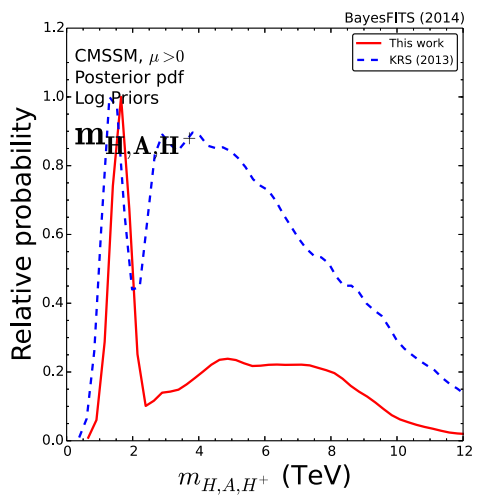

(b)

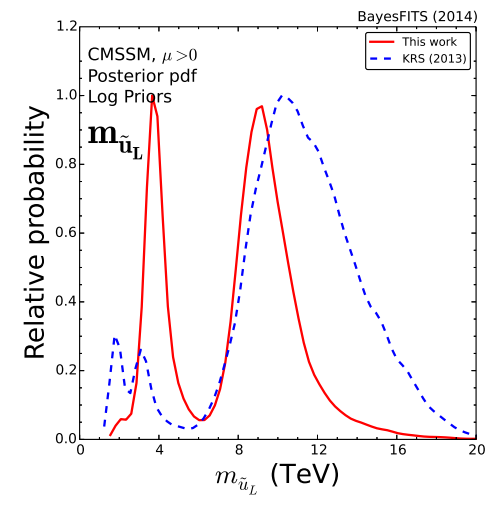

(d)

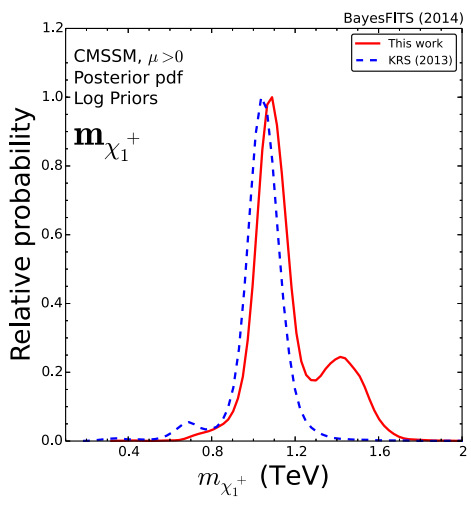

(f)

Figure 5. Marginalized 1D pdf for the heavy Higgs bosons and a selection of superpartner masses in the the CMSSM with $\mu>0$. Red solid line is this work, blue dashed lines represent distributions obtained in KRS 2013. 
range $0.9 \mathrm{TeV} \lesssim m_{\chi_{1}^{ \pm}} \lesssim 1.3 \mathrm{TeV}$. To the left and to the right of this larger mode, one can see the modes for the stau-coannihilation and $A$-resonance region, respectively. In both of them, the neutralino is bino-like, the chargino wino-like, and one finds $m_{\chi_{1}^{ \pm}} \approx 2 m_{\chi}$.

An ATLAS study [97] of the sensitivity reach for direct SUSY searches at the LHC $14 \mathrm{TeV}$ run showed that the chances of probing in this way squark masses typical of the $A$-resonance region are scant, to say the least. For example, with $3000 \mathrm{fb}^{-1}$ of integrated luminosity, 0- and 1-lepton searches for third generation squarks have the potential, when combined, to exclude at the $95 \%$ C.L. simplified models with stop next-to-LSP (NLSP) up to $m_{\tilde{t}_{1}} \simeq 1.4-1.5 \mathrm{TeV}$ for $m_{\chi} \lesssim 0.6 \mathrm{TeV}$. However, it was shown in [98] that if the same luminosity were obtained at a future $33 \mathrm{TeV}$ proton collider, one could exclude at the $95 \%$ C.L. simplified models with gluino NLSP up to $m_{\tilde{g}} \simeq 5 \mathrm{TeV}$ for $m_{\chi} \lesssim 2 \mathrm{TeV}$, or first-two generation squark NLSP up to $m_{\tilde{u}_{L}} \simeq 3.5 \mathrm{TeV}$ for $m_{\chi} \lesssim 1 \mathrm{TeV}$.

The reach for the CMSSM is going to be reduced with respect to the simplified models, due to complex decay chains that include intermediate charginos and neutralinos. Nevertheless, the same paper [98] also showed that $3000 \mathrm{fb}^{-1}$ in a $100 \mathrm{TeV}$ machine can extend the sensitivity for gluino NLSP to $m_{\tilde{g}} \simeq 12 \mathrm{TeV}$ for $m_{\chi} \lesssim 4 \mathrm{TeV}$, or first-two generation squark NLSP up to $m_{\tilde{u}_{L}} \simeq 8 \mathrm{TeV}$ for $m_{\chi} \lesssim 2 \mathrm{TeV}$.

Obviously, it seems that the $\sim 1 \mathrm{TeV}$ higgsino region of the CMSSM remains for the most part beyond the direct reach of conceivable future colliders. Note, however, that it was shown in [33] that this is not necessarily the case when the assumptions of GUT-scale universality are relaxed.

For $\mu<0$ the 1D distributions of particle masses share identical features with the positive $\mu$ case with the exception that the peaks associated with the $A$-resonance region described above are absent. Since this generally leaves only posterior probability in the region of large superpartner masses far beyond the reach of the LHC we do not show these distributions.

In figure $6(\mathrm{a})$ we show the marginalized $2 \mathrm{D}$ pdf projected to the $\left(m_{A}, \tan \beta\right)$ plane. In the $A$-resonance region, characterized by $m_{A}<2.5 \mathrm{TeV}$ and $\tan \beta>45$, the posterior appears to be well localized. In the CMSSM, this limited range of $m_{A}$ and $\tan \beta$ values is a proper feature of the $A$-resonance region, as was extensively explained in [16], namely large $\tan \beta$ value and neutralino not excessively heavy must conspire to produce a region of resonance, $m_{A} \approx 2 m_{\chi}$, and a cross section large enough to yield the relic density measured at PLANCK. One can see that $m_{A}$ and $\tan \beta$ are not as tightly constrained for the two remaining modes, so that the posterior spreads over a broad range of values. In particular, the stau-coannihilation region is localized in $m_{A}, m_{A} \simeq 1.5-2 \mathrm{TeV}$, but not in $\tan \beta$; the $\sim 1 \mathrm{TeV}$ higgsino region covers instead the majority of the parameter space with $m_{A} \gtrsim 2.5 \mathrm{TeV}, \tan \beta \gtrsim 5$. To make this feature more visible we superimposed in the figure a sample of points drawn from the posterior distribution.

It was shown in [16] that, since the points of the $A$-resonance region are well localized in the $\left(m_{A}, \tan \beta\right)$ plane, the whole region can be easily tested through precise measurement of $\operatorname{BR}\left(\mathrm{B}_{\mathrm{s}} \rightarrow \mu^{+} \mu^{-}\right)$, which is proportional to $\tan ^{6} \beta / m_{A}^{4}$. Here, we show that the same features also make a large fraction of the points testable at the LHC through Higgs searches in the $\tau^{+} \tau^{-}$channel. 


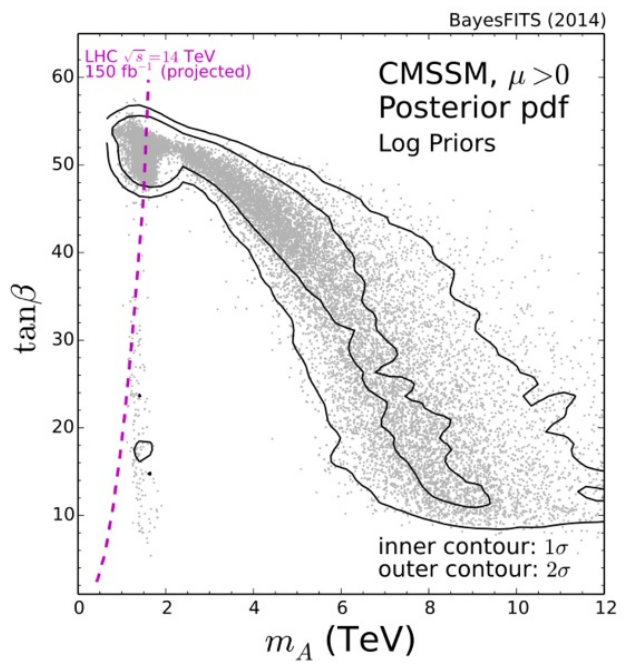

(a)

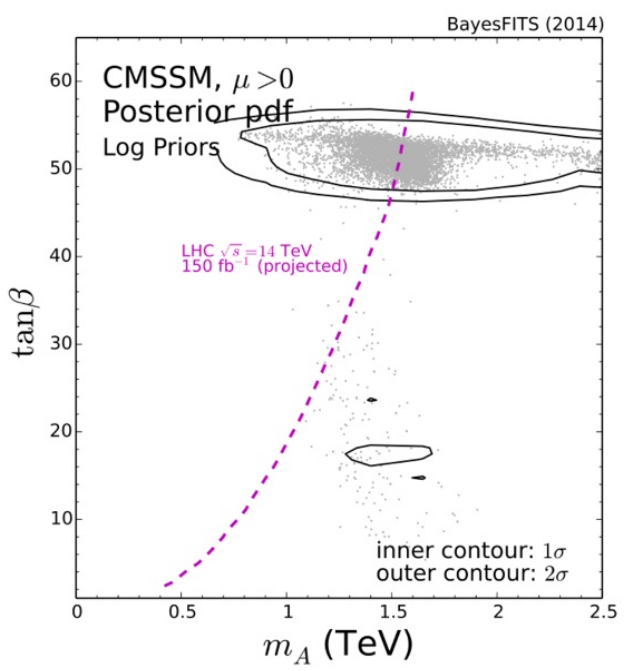

(b)

Figure 6. (a) Marginalized 2D posterior distribution for the CMSSM with $\mu>0$ in the $\left(m_{A}\right.$, $\tan \beta$ ) plane. (b) A zoomed-in fraction of the same. The inner contours give the $68 \%$ credible regions and the outer ones the $95 \%$ credible regions. A sample of points (in gray) drawn from the posterior distribution is shown for clarity. The magenta dashed lines show the expected reach at LHC $14 \mathrm{TeV}$ with $\sim 150 \mathrm{fb}^{-1}$ estimated in [99].

In figure $6(\mathrm{~b})$ we show a zoomed-in fraction of the $\left(m_{A}, \tan \beta\right)$ plane. The limits from direct $A \rightarrow \tau^{+} \tau^{-}$searches at CMS with $\sim 17 \mathrm{fb}^{-1}$ [100] are included in HiggsBounds. In the $M_{h}^{\max }$ scenario [101], they exclude $m_{A}$ up to approximately $800 \mathrm{GeV}$ for $\tan \beta \simeq 50$. However, it was explained in [102] that they are robust against radiative corrections to the MSSM Higgs, and can be considered almost scenario-independent. Ref. [99] estimated the sensitivity reach of the LHC $14 \mathrm{TeV}$ run with $\sim 150 \mathrm{fb}^{-1}$ in the $\tau^{+} \tau^{-}$channel for the heavy MSSM Higgs bosons. We show the expected 95\% C.L. reach as a magenta dashed line in figures 6(a) and 6(b). Approximately $50 \%$ of the points in the $A$-resonance region fall within the expected sensitivity.

\subsection{Prospects for dark matter detection}

In figure $7(\mathrm{a})$ we show the $2 \mathrm{D}$ posterior distribution in the $\left(m_{\chi}, \sigma_{p}^{\mathrm{SI}}\right)$ plane for $\mu>0$. The different regions are well separated and can be identified from left to right as the staucoannihilation, $A$-resonance and $\sim 1 \mathrm{TeV}$ higgsino regions. We show the current best upper limit from LUX 90\% C.L. as a red solid line, the previous one from XENON100 as a gray dot-dashed line, and the projected sensitivity of XENON-1T [103] as a magenta dashed line. The bino-like neutralino typical of the stau-coannihilation and $A$-resonance regions has a suppressed coupling to the nucleus, so that both regions lie well below the current LUX bound and it is very unlikely they will be tested, even with the improved sensitivity of XENON-1T. In contrast, the $\sim 1 \mathrm{TeV}$ higgsino region lies almost entirely within the projected XENON-1T sensitivity. The entire $68 \%$ and nearly all of the $95 \%$ credibility 


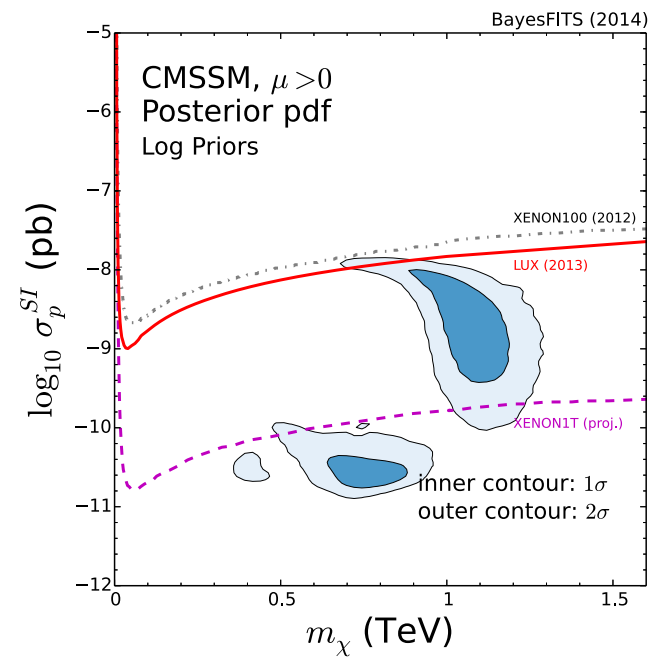

(a)

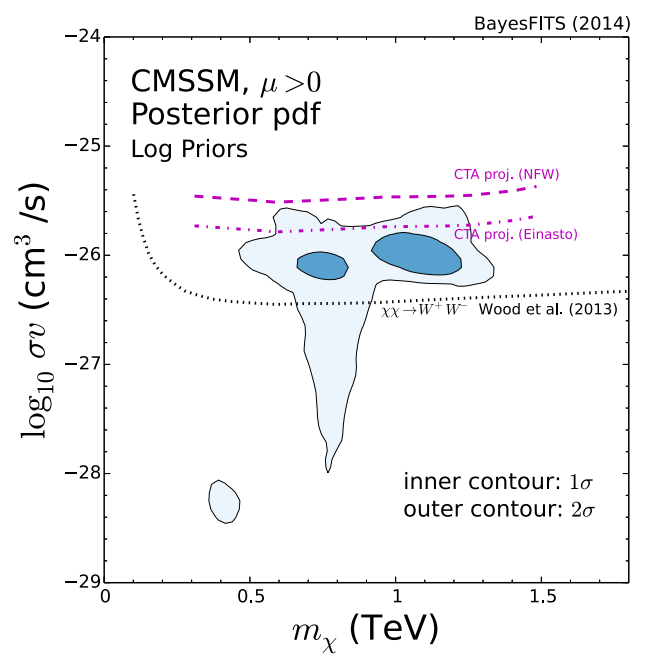

(b)

Figure 7. (a) Marginalized 2D posterior distribution for the CMSSM with $\mu>0$ in the $\left(m_{\chi}, \sigma_{p}^{\text {SI }}\right)$ plane. The red solid line shows the $90 \%$ C.L. upper bound as given by LUX, here included in the likelihood function. The gray dot-dashed line shows the 2012 XENON100 90\% C.L. bound [70] and the magenta dashed line shows projected sensitivity for 2017 at XENON-1T [103]. (b) Marginalized 2D posterior distribution for the CMSSM with $\mu>0$ in the $\left(m_{\chi}, \sigma v\right)$ plane. The magenta dashed line shows the expected sensitivity of CTA under the assumptions of [36] for a NFW halo profile. The magenta dot-dashed line shows the corresponding sensitivity with Einasto profile. The dotted black line shows the projected sensitivity of the CTA expansion considered in [104].

region have the potential to be probed in the next few years, encompassing about $70 \%$ of the points in the scan. This makes dark matter direct detection searches the predominant tool for exploration of the CMSSM.

In the CMSSM the largest cross section values, $\sigma_{p}^{\mathrm{SI}} \gtrsim 10^{-8} \mathrm{pb}$, are obtained in the focus point region. One can see the beginning of the horizontal branch joining the higgsino and focus point regions, at $m_{\chi} \simeq 0.7-0.8 \mathrm{TeV}$. The effect of the LUX limit in the likelihood is visible, as the credibility region is cut off rapidly after crossing the $90 \%$ C.L. bound, shown in red. In contrast to [16], this causes the focus point region to be disfavored by the scan. In the $\mu<0$ scenario we obtain the same results albeit with the absence of the $A$-resonance region. The sign of the $\mu$ parameter has little impact on $\sigma_{p}^{\mathrm{SI}}$ for the neutralino and the $\sim 1 \mathrm{TeV}$ higgsino region with $\mu<0$ can also be entirely probed by XENON-1T.

In figure $7(\mathrm{~b})$ we show the $2 \mathrm{D}$ posterior distribution in the $\left(m_{\chi}, \sigma v\right)$ plane. The node at $\sigma v \lesssim 10^{-28} \mathrm{~cm}^{3} / \mathrm{s}$ is the stau-coannihilation region, which has a much reduced $\sigma v$ in the present day due to the absence of co-annihilations with the stau NLSP, which are instead only present in the early Universe. The $A$-resonance and $\sim 1 \mathrm{TeV}$ higgsino regions are visible at larger $\sigma v$, from left to right, respectively. The $A$-resonance region is characterized by a broad range of cross section values, with a deep funnel at $95 \%$ credibility that extends down to $\sigma v \simeq 10^{-28} \mathrm{~cm}^{3} / \mathrm{s}$. This corresponds to a large resonant effect in the 
early Universe when the neutralinos are distributed thermally, but the present value of $\sigma v$ is small since the colliding neutralinos have insufficient energy to produce the pseudoscalar on shell (see, e.g., appendix B in [31]). $\sigma v$ is reduced by orders of magnitude in this funnel and is effectively impossible to probe via indirect detection.

As was the case for direct detection, the $\sim 1 \mathrm{TeV}$ higgsino region presents a particularly promising target for indirect detection since the annihilation cross section is restricted to a small range close to the thermal value compared to other dark matter candidates in the MSSM which can have much lower annihilation cross sections. The magenta dashed and dot-dashed lines in figure 7(b) show the expected sensitivity of CTA derived in section 2 under the assumptions of the NFW [105] and Einasto [106] halo profile, respectively. The dotted line shows the projected sensitivity of CTA to the $W^{+} W^{-}$final state, as obtained in ref. [104] under a more optimistic setup including more telescopes in the array. As previously discussed, limits on single final states may not automatically exclude all points in the posterior since the neutralino will usually annihilate into several different final states depending on its mass and composition. The $W^{+} W^{-}$final state does however provide a good approximation in the $\sim 1 \mathrm{TeV}$ higgsino region and points in this region lying above the $W^{+} W^{-}$line have the potential to be constrained. We note that current limits from the Cherenkov telescope array HESS [83] are approximately an order of magnitude larger than the projected CTA sensitivity and so do not constrain the posterior regions found.

One can see that the derived CTA limits lie just above above the $68 \%$ credibility posterior region for either choice of dark matter profile, but significant improvements to the limits are possible by improving the experimental setup. It should be noted that a factor of five improvement in the model independent limit on the number of observed gamma rays would be sensitive to the entire $\sim 1 \mathrm{TeV}$ higgsino region and to the bulk of the $A$-resonance region, thus the vast majority of the favored points in the CMSSM.

The higgsino and stau-coannihilation regions in the negative $\mu$ case share the same properties as the positive $\mu$ case and have the same prospects for detection, we therefore do not show their distribution here.

\section{Results in the NUHM}

\subsection{Posterior distributions and prospects for collider searches}

We proceed now to the analysis of the NUHM. The parameters $m_{0}, m_{1 / 2}, A_{0}$, and $\tan \beta$ were scanned in the same ranges as in the CMSSM. The parameters $m_{H_{d}}^{2}$ and $m_{H_{u}}^{2}$ were allowed to assume negative values at the GUT scale; see table 2. We limit ourself to the case with $\mu>0$, which, as was seen for the CMSSM, presents a greater number of solutions. One must keep in mind that the solutions with $\mu<0$ can generally be mapped to a subset of the ones we present in here, without novel phenomenological features.

In figure $8(\mathrm{a})$ we show the marginalized $2 \mathrm{D}$ posterior in the $\left(m_{0}, m_{1 / 2}\right)$ plane. The $68 \%$ and $95 \%$ credible regions are indicated by inner and outer solid contours, respectively. In this and the following figures we superimpose on the clearly marked credible regions a set of points drawn from the posterior distribution. We include those points since, unlike in the CMSSM, the points that satisfy the relic density constraint are not always found in regions 


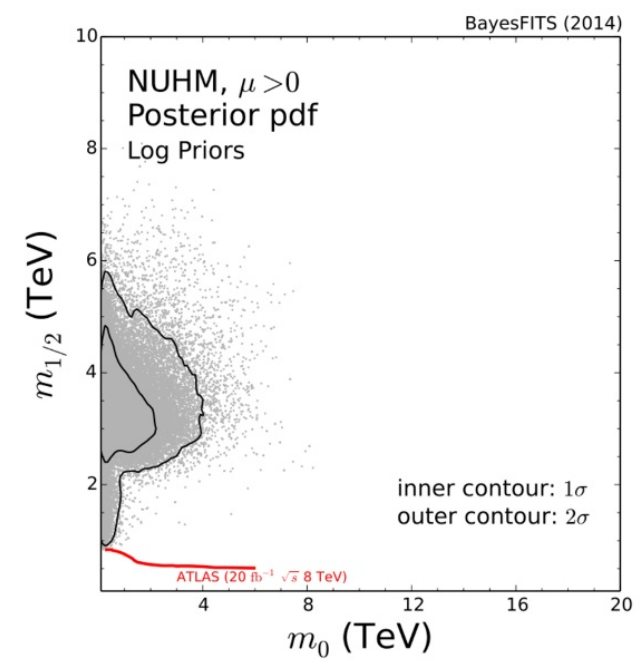

(a)

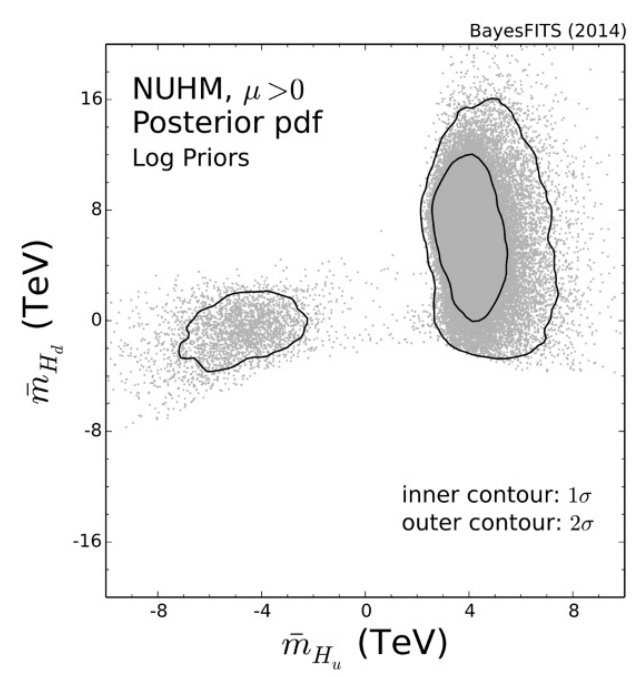

(c)

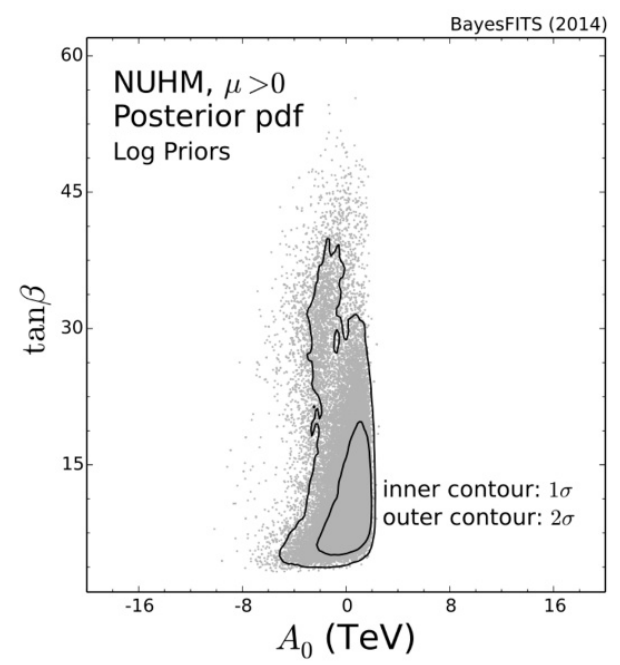

(b)

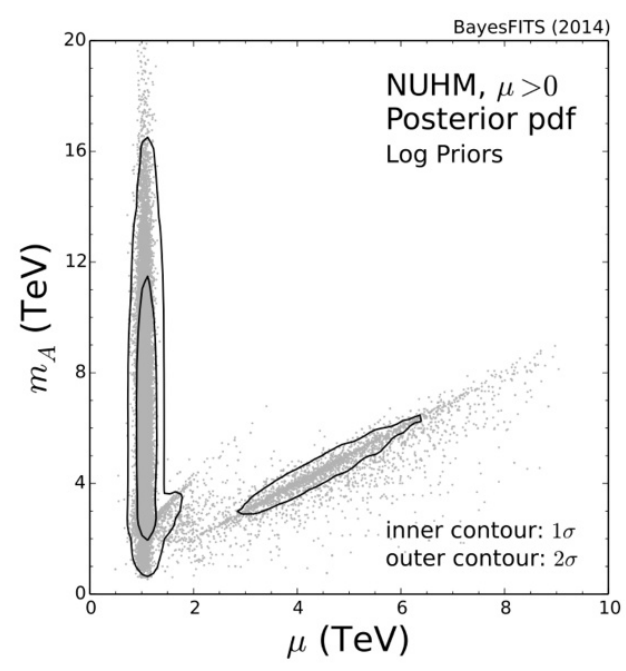

(d)

Figure 8. Marginalized 2D posterior in (a) the $\left(m_{0}, m_{1 / 2}\right)$, (b) the $\left(A_{0}, \tan \beta\right)$, (c) the $\left(\bar{m}_{H_{u}}\right.$, $\bar{m}_{H_{d}}$ ), and (d) the $\left(\mu, m_{A}\right)$ planes of the NUHM with $\mu>0.68 \%$ and $95 \%$ credible regions are shown by the inner and outer contours, respectively. Gray points are distributed according to the posterior probability. The ATLAS 95\% C.L. exclusion line is shown in red solid for reference. 
of parameter space well separated along different mechanisms of neutralino annihilation. Thus, the distribution and position of the points in the plots highlight details not always easy to infer from the contours and help indicate where additional solutions beyond the $95 \%$ credibility regions can be found.

The mechanisms to reduce the relic density are for the most part in common with the CMSSM: stau co-annihilation and $A$-resonance of bino-like neutralinos, and annihilation and co-annihilation of $\sim 1 \mathrm{TeV}$ higgsino-like neutralinos and charginos. We shall see, though, that the NUHM also presents some additional ways of obtaining the correct relic density. Moreover, all of those mechanisms can be obtained for $m_{0}$ not too large, in contrast with the $\sim 1 \mathrm{TeV}$ higgsino region of the CMSSM, thanks to the additional freedom in the Higgs sector. Thus the posterior shows a more compact shape in $\left(m_{0}, m_{1 / 2}\right)$ plane, due also to the effect of log prior distributions. Had we used flat priors, volume effects would inflate the region of large $m_{0}$ and $m_{1 / 2}$ and therefore increase the importance of the $\sim 1 \mathrm{TeV}$ higgsino region in the NUHM.

The marginalized posterior in the $\left(A_{0}, \tan \beta\right)$ plane is shown in figure $8(\mathrm{~b})$. As was the case for the CMSSM, the solutions tend to be distributed over the entire $\tan \beta$ range, but now favor relatively smaller values of $\left|A_{0}\right|$ than in previous scans.

In figure $8(\mathrm{c})$ we show the marginalized $2 \mathrm{D}$ pdf in the $\left(\bar{m}_{H_{u}}, \bar{m}_{H_{d}}\right)$ plane. ${ }^{4}$ One can see here a previously unexplored $95 \%$ credibility region featuring negative values for $\bar{m}_{H_{u}}$ at the GUT scale and, for the majority of the points involved, also $\bar{m}_{H_{d}}<0$.

Large negative values of $m_{H_{u}}^{2}$ at the GUT scale lead to large negative value for the same parameter at $M_{\mathrm{SUSY}}$. Through the EWSB condition, these points thus feature very large values of $\mu$, up to ranges previously unexplored in NUHM analyses. We show the marginalized $2 \mathrm{D}$ pdf in the $\left(\mu, m_{A}\right)$ plane in figure $8(\mathrm{~d})$. The described solutions can be seen on the lower right end of the plot, for values of $\mu$ that can be as large as $9 \mathrm{TeV} .{ }^{5}$

The correct relic density for these points is obtained through mechanisms of staucoannihilation. In fact, values of $\mu$ so large strongly enhance the coupling of the lightest stau to the $H_{u}$ component of the lightest Higgs, thus increasing the efficiency of the annihilation channel $\tilde{\tau} \tilde{\tau} \rightarrow h h$. The $\tilde{\tau} \tilde{\tau} h$ vertex, proportional to $\mu$, leads to a $\mu^{4}$ enhancement of the annihilation cross section, which becomes the dominant mechanism when the lightest stau and neutralino are almost degenerate in mass. We show in figure $9(\mathrm{a})$ the $2 \mathrm{D}$ pdf in the $\left(m_{\chi}, m_{\tilde{\tau}_{1}}\right)$ plane. One can see that because of the above considerations, stau-coannihilation in the NUHM is efficient up to $m_{\chi} \simeq 2 \mathrm{TeV}$, thus significantly extending the range observed in the CMSSM, or in previous studies performed with more limited ranges of $\mu[11,14]$.

Figures $8(\mathrm{c})$ and $8(\mathrm{~d})$ show that the vast majority of the points with high posterior probability feature, at the GUT scale, positive values of $m_{H_{u}}^{2}$ and $m_{H_{d}}^{2}$, and at the weak scale, $\mu \simeq 1 \mathrm{TeV}$. Thus, as was the case for the CMSSM, in the NUHM the largest number

\footnotetext{
${ }^{4}$ Here $\bar{m}_{H_{u}}$ and $\bar{m}_{H_{d}}$ refer to the signed square root of the absolute value of $m_{H_{u}}^{2}$ and $m_{H_{d}}^{2}$, respectively. E.g., $\bar{m}_{H_{u}}=m_{H_{u}}^{2} / \sqrt{\left|m_{H_{u}}^{2}\right|}$.

${ }^{5}$ It is clear that these solutions present uncomfortably large values of EW fine tuning due the large $\mu$ parameter, so that they might be unappealing from a theoretical point of view. However, in this paper we are only concerned with the existence of viable phenomenological solutions, independently of theoretical considerations. We will therefore treat these solutions without further mentioning the fine-tuning issue.
} 


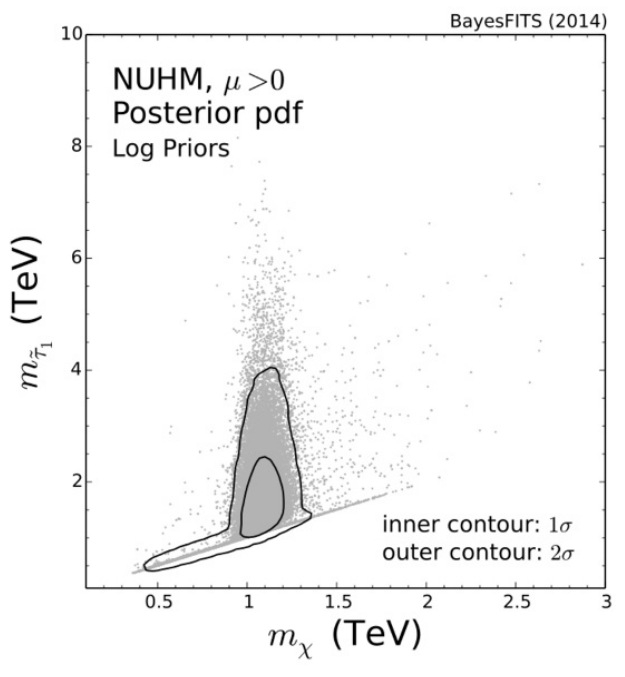

(a)

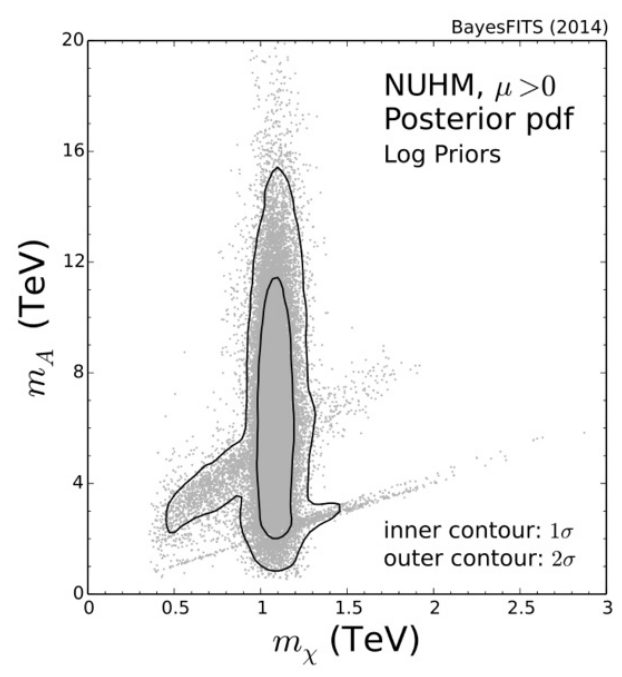

(b)

Figure 9. Marginalized 2D posterior in (a) the $\left(m_{\chi}, m_{\tilde{\tau}_{1}}\right)$ plane and (b) the $\left(m_{\chi}, m_{A}\right)$ plane of the NUHM with $\mu>0.68 \%$ and $95 \%$ credible regions are shown by the inner and outer contours, respectively. Gray points are distributed according to the posterior probability.

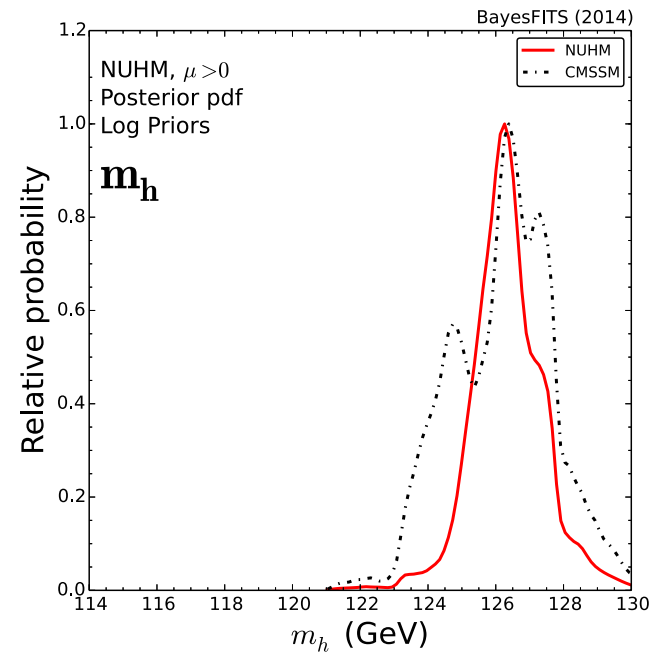

Figure 10. Marginalized 1D pdf of $m_{h}$ for the NUHM with $\mu>0$. The black dot-dashed line shows the distribution obtained for the CMSSM with $\mu>0$ for comparison. 
of solutions belong to the $\sim 1 \mathrm{TeV}$ higgsino region. The predominance of this region was also shown in ref. [14], where it was the only region found, due to their choice of input parameters and prior ranges. The extended stau-coannihilation region shown in figures 8 and 9 is instead a novel finding of this study.

Moreover, as was mentioned at the beginning of this section, in the NUHM there are also a significant number of solutions for which the neutralino annihilates via the resonance with heavy Higgs bosons, as already pointed out in $[16,30]$. We show in figure $9(\mathrm{~b})$ the $2 \mathrm{D}$ pdf in the $\left(m_{\chi}, m_{A}\right)$ plane, which shows the presence of many points at $m_{A} \approx 2 m_{\chi}$. Those points cannot be identified as easily in other plots: for example, in figure 8(c) they are shown as diffuse points lying between the two high posterior probability modes described above, and as a subset of points in the right hand mode restricted to $\bar{m}_{H_{d}}<4 \mathrm{TeV}$ (above this value $m_{H_{d}}^{2}$ drives $m_{A}$ to be too large to find solutions with $m_{A} \approx 2 m_{\chi}$ ). We point out here that, while in the CMSSM the points of the $A$-resonance region feature neutralinos with very large bino composition, and very large $\tan \beta$ values, this is not always the case in the NUHM. Thus, while in the CMSSM the main annihilation channel for those points is $\chi \chi \rightarrow b \bar{b}$, many of the $A$-resonance points with $m_{\chi}>1.2 \mathrm{TeV}$ in figure 9 (b) feature much larger higgsino fraction and moderate $\tan \beta$ values, so that the correct relic density is obtained in a combined fashion: with $t \bar{t}$ final states in addition to $b \bar{b}$, and through resonance with the heavy scalar $H$, degenerate with $A$, in addition to the pseudoscalar. As we shall see in section 4.2 this fact has important consequences for dark matter direct detection.

Figure $9(\mathrm{~b})$ neatly shows the different annihilation mechanisms described above: the large concentration of points at $m_{\chi} \simeq 1 \mathrm{TeV}$ gives the $\sim 1 \mathrm{TeV}$ higgsino region; at $m_{A} \approx$ $2 m_{\chi}$ there is the strip of points belonging to the just-described $A / H$-resonance region, which induces a lobe in the $95 \%$ credible region of the posterior for $m_{\chi} \gtrsim 1.2 \mathrm{TeV}$; and, finally, the stau-coannihilation region, which produces a lobe in the posterior with $m_{\chi} \lesssim$ $1 \mathrm{TeV}$, but can in fact extend up to $m_{\chi} \lesssim 2 \mathrm{TeV}$.

We show in figure 10 the 1D pdf distribution of the lightest Higgs mass, $m_{h}$. The black dot-dashed line shows the distribution obtained for the CMSSM with $\mu>0$ for comparison. One can see that the posterior is prevalently uni-modal due to the statistical dominance of the $\sim 1 \mathrm{TeV}$ higgsino region in the NUHM.

In figure 11 we present the marginalized 1D posterior distributions for the heavy Higgs bosons and a selection of superpartner masses. As a comparison we show the corresponding CMSSM distributions from figure 5 as black dot-dashed lines. Figure 11(a) shows the distribution for $m_{\chi}$. One can see one single peak at $m_{\chi} \simeq 1 \mathrm{TeV}$, indicating that in the NUHM the $\sim 1 \mathrm{TeV}$ higgsino region dominates the posterior probability, with the other two regions significantly less favored and spread over a broad range of $m_{\chi}$ values.

In figure 11(b) we show the distribution of the heavy Higgs masses. As mentioned above, the $A / H$-resonance region extends over a large range of $m_{A}$ values, and the posterior probability associated with it is much reduced relative to the CMSSM. As a consequence, values of $m_{A}>2-3 \mathrm{TeV}$, typical of the $\sim 1 \mathrm{TeV}$ higgsino region, are instead favored in the NUHM, thus making the prospects for sensitivity at the LHC $\left(A \rightarrow \tau^{+} \tau^{-}\right.$direct searches $)$ much bleaker than in the CMSSM. 


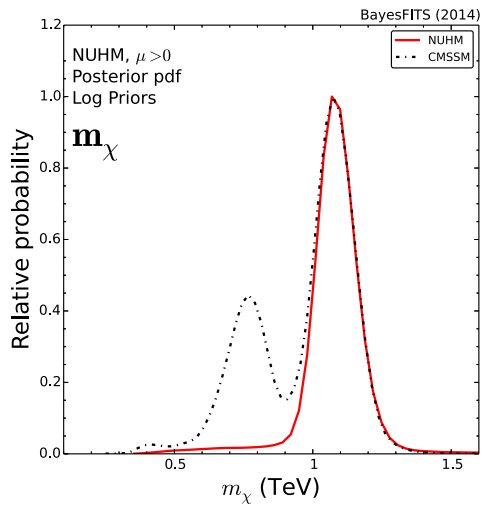

(a)

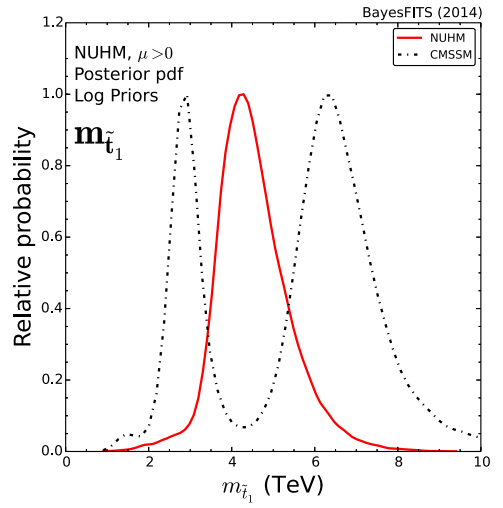

(c)

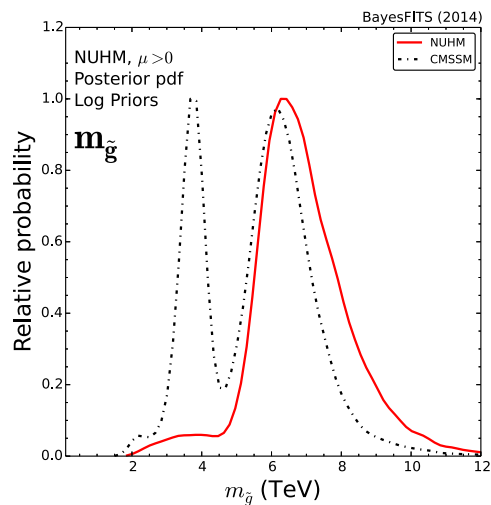

(e)

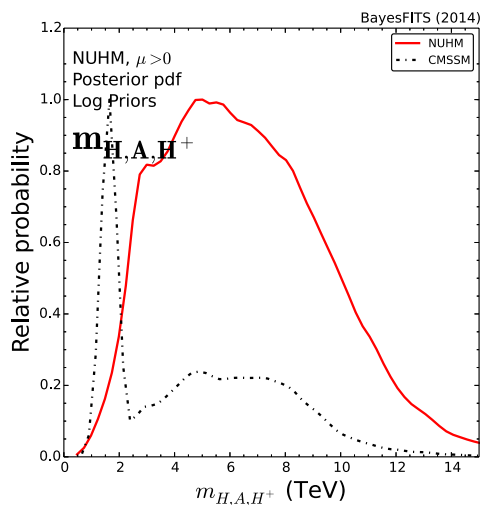

(b)

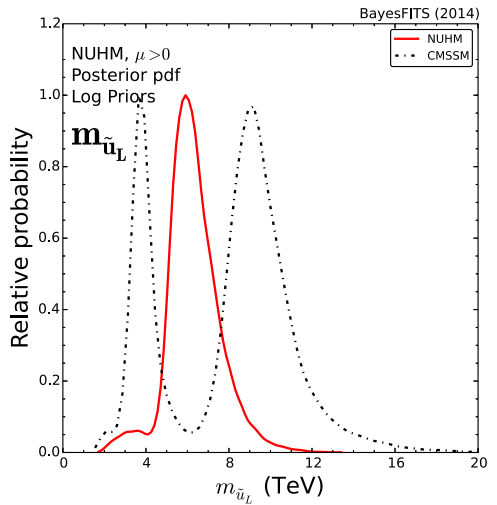

(d)

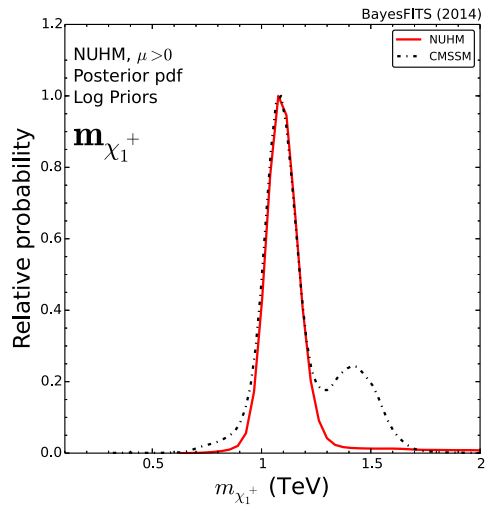

(f)

Figure 11. Marginalized 1D pdf for the heavy Higgs bosons and a selection of superpartner masses in the NUHM with $\mu>0$. Black dot-dashed lines are the distributions obtained for the CMSSM with $\mu>0$ for comparison. 
Analogous conclusions pertain to the prospects for LHC observation of the other SUSY particles. In figures $11(\mathrm{c})$ and $11(\mathrm{~d})$ we show the distributions for the lightest stop mass and squark masses for the first two generations, respectively. In figure 11(e) we show the distribution for the gluino mass. The bulk of the squark mass distributions are peaked around mass values significantly smaller than in the corresponding $\sim 1 \mathrm{TeV}$ higgsino region of the CMSSM, as the posterior does not extend as much in $m_{0}$, but they are still well outside the most optimistic reach for direct detection at the LHC.

One observes some solutions in common with the CMSSM, in the stau-coannihilation region, characterized by $m_{\tilde{t}_{1}} \lesssim 1.5 \mathrm{TeV}, m_{\tilde{u}_{L}} \lesssim 3 \mathrm{TeV}$, or $m_{\tilde{g}} \lesssim 3 \mathrm{TeV}$, and a neutralino that can be as light as $0.4 \mathrm{TeV}$. Those might begin to be probed at the $14 \mathrm{TeV}$ run of the LHC. However, as was explained above, the stau-coannihilation region in the NUHM extends significantly with respect to the CMSSM, reaching quite large $m_{1 / 2}$ values. Thus, it favors heavier gluinos, neutralinos, and scalars, and the statistical weight of the parameter space in reach of the LHC is much reduced.

Finally, we show for completeness in figure 11(f) the 1D pdf for the lightest chargino. One can see the predominant peak at $m_{\chi_{1}^{ \pm}} \simeq 1 \mathrm{TeV}$, encompassing models with higgsinolike $\chi_{1}^{ \pm}$, accompanied by a lower tail that extends to larger mass values, typical of the wino-dominated charginos.

\subsection{Prospects for dark matter detection}

In figure 12(a) we show the marginalized 2D posterior distribution in the $\left(m_{\chi}, \sigma_{p}^{\mathrm{SI}}\right)$ plane. As was the case in the CMSSM, shown in figure 7(a), one can easily identify the $\sim 1 \mathrm{TeV}$ higgsino region as the large $68 \%$ and $95 \%$ credible region at $m_{\chi} \simeq 1-1.2 \mathrm{TeV}$ right below the LUX limit.

The characteristics of this region are largely independent of the model, so that the prospects for detection are similar to the CMSSM. However, the relative probability of this region is larger in the NUHM, being greater than $90 \%$, versus approximately $70 \%$ of the total probability in the same region of the CMSSM.

On top of this, as was mentioned when discussing figure 9(b), many of the solutions in the $A / H$-resonance region of the NUHM feature mixed composition, bino-higgsino neutralinos with $m_{\chi} \gtrsim 1.2 \mathrm{TeV}$, with consequently enhanced couplings to the nucleus. Those points can be seen in figure 12(a) scattered below the LUX limit, well in reach of the XENON-1T sensitivity, shown with a magenta dashed line,

On the negative side, one can see that the remaining $95 \%$ credible region, the staucoannihilation region, now extends to much smaller values of $\sigma_{p}^{\mathrm{SI}}$ and for neutralinos heavier than $m_{\chi} \simeq 0.8 \mathrm{TeV}$ it lies below the onset of irreducible neutrino background $[107,108]$ calculated in [109], shown with a black dotted line. For $\sigma_{p}^{\text {SI }}$ below the black dotted line the background of atmospheric and diffuse supernova neutrinos becomes important, so that the sensitivity scales with square root of exposure, making the neutralino more difficult to detect via direct detection experiments.

Finally, we show in figure 12(b) 2D posterior distribution in the $\left(m_{\chi}, \sigma v\right)$ plane. As was explained in section 2 the estimated CTA sensitivity is indicatively shown for the NFW 


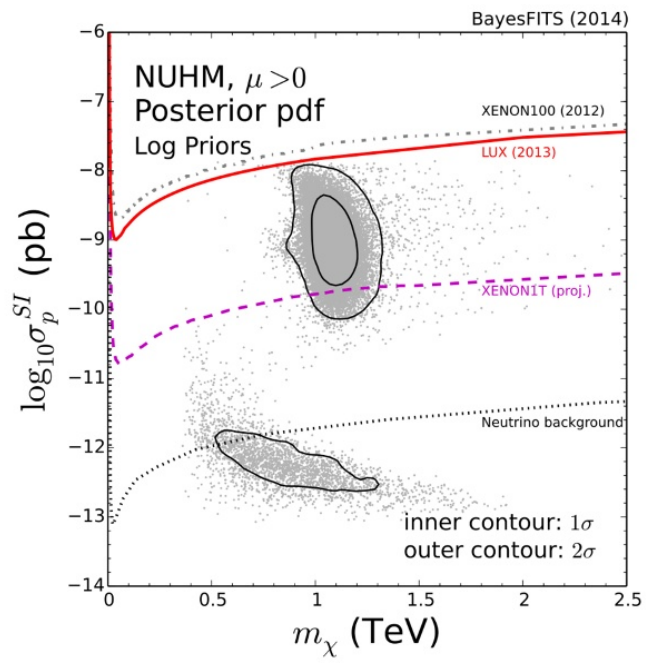

(a)

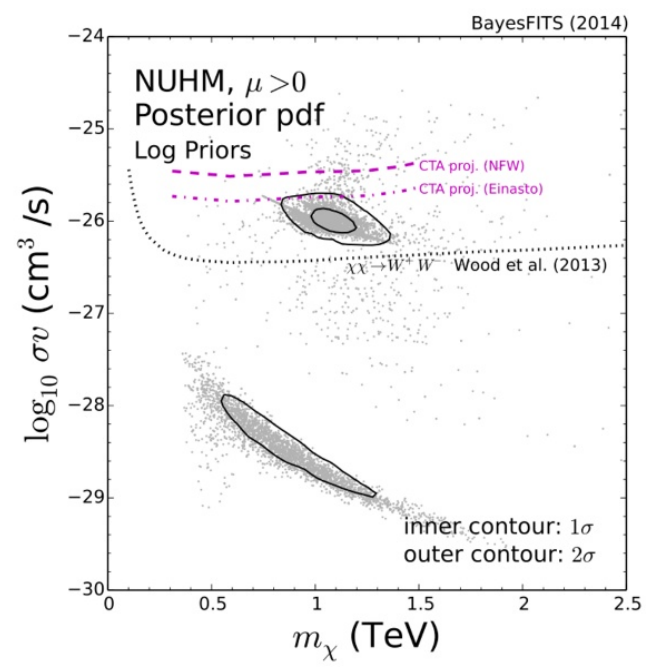

(b)

Figure 12. (a) Marginalized 2D posterior distribution in the $\left(m_{\chi}, \sigma_{p}^{\mathrm{SI}}\right)$ plane of the NUHM with $\mu>0$. The solid red line shows the $90 \%$ C.L. upper bound as given by LUX, here included in the likelihood function. The dot-dashed gray line shows the 90\% C.L. 2012 bound of XENON100. The projected sensitivity for 2017 at XENON-1T is shown in magenta dashed. The black dotted line marks the onset of the irreducible neutrino background. (b) Marginalized 2D posterior distribution for the NUHM with $\mu>0$ in the $\left(m_{\chi}, \sigma v\right)$ plane. The magenta dashed line shows the expected sensitivity of CTA under the assumption of a NFW halo profile. The magenta dot-dashed line shows the corresponding sensitivity with Einasto profile. The thin dotted line shows the projected sensitivity of the CTA expansion [104].

and Einasto halo profile as a magenta dashed and dot-dashed line, respectively. One must remember that the sensitivity of the individual points in the scan strongly depends on the annihilation final states, so that the lines in figure 12(b) must be taken with care. However, the indicated sensitivity is robust for final states characterized by gauge bosons, which are very typical of the $\sim 1 \mathrm{TeV}$ higgsino region, shown in the figure as the predominant $68 \%$ and $95 \%$ posterior region.

Thus, one can extend to the NUHM the conclusion already stated for the CMSSM in section 3.2. The projected sensitivity in the minimal configuration studied here ${ }^{6}$ seems to fall just short of biting significantly into the parameter space of the model. But extended configurations considered in the literature or, alternatively, an improvement in the estimated sensitivity, have the potential to deeply probe the bulk of the model's parameter space, thus yielding a complementary test with respect to direct detection searches.

\footnotetext{
${ }^{6}$ We use the setup considered in [36] called Array I consisting of 3 large size telescopes, 18 medium size telescopes and 56 small size telescopes.
} 


\section{Summary and conclusions}

In this paper we performed a Bayesian analysis of the CMSSM and the NUHM. We presented the $68 \%$ and $95 \%$ credible regions of the marginalized $2 \mathrm{D}$ posterior pdf and the 1D distributions of relevant parameters and observables in light of the latest experimental constraints and updated numerical tools.

In particular, we updated the results of our previous study [16] by a) including the corrections to the lightest Higgs mass beyond the 2-loop order using FeynHiggs v2.10.0, which calculates the leading and next-to-leading log corrections in the top/stop sector resummed at all orders in perturbation theory; $b$ ) including in the likelihood function the latest constraints from direct SUSY searches at the LHC with $\sim 20 \mathrm{fb}^{-1}$ at $8 \mathrm{TeV}$; and $c$ ) including in the likelihood function the most recent constraints from direct detection of dark matter at LUX.

We find that the higher-order corrections to the Higgs mass induce modifications to the posterior probability distribution with respect to [16]. The correct value of the Higgs boson mass now requires an $M_{\text {SUSY }}$ in general lower, so that the $95 \%$ credible regions do not extend beyond $m_{0} \simeq 12 \mathrm{TeV}$ in the CMSSM. Moreover, regions of the parameter space that in the past struggled to produce a $\sim 126 \mathrm{GeV}$ Higgs mass, can now do it more easily. As a consequence, we observe increased statistical relevance of the $A$-resonance region of the CMSSM relative to [16], with approximately $30 \%$ of the total probability. This improves the chances for direct observations of the pseudoscalar Higgs at the LHC $14 \mathrm{TeV}$ run, in the $A \rightarrow \tau^{+} \tau^{-}$channel. It also potentially favors direct testing in sparticle searches at future, higher-energy proton colliders.

On the other hand, the bulk of the probability still lies in the $\sim 1 \mathrm{TeV}$ higgsino region, comprising $\sim 70 \%$ of the total in the CMSSM and $\sim 90 \%$ in the NUHM. Given the almost pure higgsino nature of the dark matter candidate in this region the prospect for probing the vast majority of the parameter space of both models through dark matter detection searches is enticing.

The constraining power of future 1-tonne direct detection experiments like XENON-1T on the parameter space of the CMSSM and the NUHM has been long known, and is confirmed once more in here. In this study we also showed that indirect detection of dark matter through $\gamma$-rays from the GC at CTA is a realistic possibility in the CMSSM and the NUHM. We applied the results of ref. [36] for the sensitivity of CTA to several annihilation final states to the case of annihilation to multiple final states, as is the MSSM. We find that the configuration studied in [36] falls just short of biting significantly into the parameter space, but a factor of 5 improvement on the sensitivity can probe $\sim 90 \%$ of the favored parameter space in both models.

In summary, in both models all the regions favored by the latest constraints show good prospects for future observation. In the CMSSM, the stau-coannihilation region (1\% of the total pdf) will be most likely probed in its entirety through direct SUSY searches at the LHC $14 \mathrm{TeV}$ run; $\sim 50 \%$ of the $A$-resonance region (corresponding to $15 \%$ of the total pdf) might be probed in Higgs searches at the LHC, while it was shown in [16] that the whole region is also very sensitive to improvements in the measurement of $\mathrm{BR}\left(\mathrm{B}_{\mathrm{s}} \rightarrow \mu^{+} \mu^{-}\right)$; the 
$\sim 1 \mathrm{TeV}$ higgsino region (70\% of the pdf) will be probed via dark matter direct detection in 1-tonne experiments. Simultaneously, optimistic but not unrealistic improvements in the projected sensitivity of CTA might potentially test the whole $\sim 1 \mathrm{TeV}$ higgsino region and almost all of the $A$-resonance region.

This becomes even more important in the NUHM, where dark matter searches become the privileged instrument to probe the parameter space. In particular the largest part of the $\sim 1 \mathrm{TeV}$ higgsino region and a large number of $A / H$ resonance solutions, that together cover approximately $\sim 90 \%$ of the favored parameter space can be simultaneously probed at 1-tonne detectors and at CTA, if the estimated sensitivity of the latter increases as shown in this study.

\section{Acknowledgments}

We would like to thank Kamila Kowalska for valuable input on the construction of the LUX likelihood. A.W. would like to thank Jamie Tattersall for email support on using CheckMATE. This work has been funded in part by the Welcome Programme of the Foundation for Polish Science. L.R. is also supported in part by a STFC consortium grant of Lancaster, Manchester, and Sheffield Universities. The use of the CIS computer cluster at the National Centre for Nuclear Research is gratefully acknowledged.

Open Access. This article is distributed under the terms of the Creative Commons Attribution License (CC-BY 4.0), which permits any use, distribution and reproduction in any medium, provided the original author(s) and source are credited.

\section{References}

[1] ATLAS collaboration, Observation of a new particle in the search for the Standard Model Higgs boson with the ATLAS detector at the LHC, Phys. Lett. B 716 (2012) 1 [arXiv: 1207.7214] [INSPIRE].

[2] CMS collaboration, Observation of a new boson at a mass of $125 \mathrm{GeV}$ with the CMS experiment at the LHC, Phys. Lett. B 716 (2012) 30 [arXiv:1207.7235] [INSPIRE].

[3] G.L. Kane, C.F. Kolda, L. Roszkowski and J.D. Wells, Study of constrained minimal supersymmetry, Phys. Rev. D 49 (1994) 6173 [hep-ph/9312272] [INSPIRE].

[4] H. Baer, V. Barger and A. Mustafayev, Implications of a $125 \mathrm{GeV}$ Higgs scalar for LHC SUSY and neutralino dark matter searches, Phys. Rev. D 85 (2012) 075010 [arXiv:1112.3017] [INSPIRE].

[5] M. Kadastik, K. Kannike, A. Racioppi and M. Raidal, Implications of the 125 GeV Higgs boson for scalar dark matter and for the CMSSM phenomenology, JHEP 05 (2012) 061 [arXiv:1112.3647] [INSPIRE].

[6] J. Cao, Z. Heng, D. Li and J.M. Yang, Current experimental constraints on the lightest Higgs boson mass in the constrained MSSM, Phys. Lett. B 710 (2012) 665 [arXiv:1112.4391] [INSPIRE].

[7] J. Ellis and K.A. Olive, Revisiting the Higgs mass and dark matter in the CMSSM, Eur. Phys. J. C 72 (2012) 2005 [arXiv:1202.3262] [INSPIRE]. 
[8] H. Baer, V. Barger and A. Mustafayev, Neutralino dark matter in mSUGRA/CMSSM with a 125 GeV light Higgs scalar, JHEP 05 (2012) 091 [arXiv: 1202.4038] [INSPIRE].

[9] P. Bechtle et al., Constrained supersymmetry after two years of LHC data: a global view with Fittino, JHEP 06 (2012) 098 [arXiv: 1204.4199] [INSPIRE].

[10] C. Balázs, A. Buckley, D. Carter, B. Farmer and M. White, Should we still believe in constrained supersymmetry?, Eur. Phys. J. C 73 (2013) 2563 [arXiv:1205.1568] [INSPIRE].

[11] A. Fowlie et al., The CMSSM favoring new territories: the impact of new LHC limits and a 125 GeV Higgs, Phys. Rev. D 86 (2012) 075010 [arXiv: 1206. 0264] [INSPIRE].

[12] S. Akula, P. Nath and G. Peim, Implications of the Higgs boson discovery for mSUGRA, Phys. Lett. B 717 (2012) 188 [arXiv:1207.1839] [INSPIRE].

[13] O. Buchmueller et al., The CMSSM and NUHM1 in light of $7 \mathrm{TeV} L H C, B_{s} \rightarrow \mu^{+} \mu^{-}$and XENON100 data, Eur. Phys. J. C 72 (2012) 2243 [arXiv:1207.7315] [INSPIRE].

[14] C. Strege et al., Global fits of the CMSSM and NUHM including the LHC Higgs discovery and new XENON100 constraints, JCAP 04 (2013) 013 [arXiv: 1212.2636] [INSPIRE].

[15] M.E. Cabrera, J.A. Casas and R.R. de Austri, The health of SUSY after the Higgs discovery and the XENON100 data, JHEP 07 (2013) 182 [arXiv:1212.4821] [INSPIRE].

[16] K. Kowalska, L. Roszkowski and E.M. Sessolo, Two ultimate tests of constrained supersymmetry, JHEP 06 (2013) 078 [arXiv: 1302.5956] [INSPIRE].

[17] A. Dighe, D. Ghosh, K.M. Patel and S. Raychaudhuri, Testing times for supersymmetry: looking under the lamp post, Int. J. Mod. Phys. A 28 (2013) 1350134 [arXiv:1303.0721] [INSPIRE].

[18] T. Cohen and J.G. Wacker, Here be dragons: the unexplored continents of the CMSSM, JHEP 09 (2013) 061 [arXiv:1305.2914] [INSPIRE].

[19] Planck collaboration, P.A.R. Ade et al., Planck 2013 results. XVI. Cosmological parameters, arXiv:1303.5076 [INSPIRE].

[20] Electroweak radiative B decays webpage, http://www.slac.stanford.edu/xorg/hfag/rare/2012/radll/index.html.

[21] Belle collaboration, I. Adachi et al., Evidence for $B^{-} \rightarrow \tau^{-} \bar{\nu}_{\tau}$ with a hadronic tagging method using the full data sample of Belle, Phys. Rev. Lett. 110 (2013) 131801 [arXiv:1208.4678] [INSPIRE].

[22] LHCb collaboration, Measurement of the $B_{s}^{0} \rightarrow \mu^{+} \mu^{-}$branching fraction and search for $B^{0} \rightarrow \mu^{+} \mu^{-}$decays at the LHCb experiment, Phys. Rev. Lett. 111 (2013) 101805 [arXiv:1307.5024] [INSPIRE].

[23] CMS collaboration, Measurement of the $B_{s} \rightarrow \mu^{+} \mu^{-}$branching fraction and search for $B^{0} \rightarrow \mu^{+} \mu^{-}$with the CMS experiment, Phys. Rev. Lett. 111 (2013) 101804 [arXiv: 1307.5025$]$ [INSPIRE].

[24] Muon G-2 collaboration, G.W. Bennett et al., Final report of the muon E821 anomalous magnetic moment measurement at BNL, Phys. Rev. D 73 (2006) 072003 [hep-ex/0602035] [INSPIRE].

[25] J.P. Miller, E. de Rafael and B.L. Roberts, Muon (g-2): experiment and theory, Rept. Prog. Phys. 70 (2007) 795 [hep-ph/0703049] [InSPIRE]. 
[26] CMS collaboration, Inclusive search for supersymmetry using the razor variables in $p p$ collisions at $\sqrt{s}=7 \mathrm{TeV}$, Phys. Rev. Lett. 111 (2013) 081802 [arXiv:1212.6961] [InSPIRE].

[27] CMS collaboration, Search for supersymmetry in hadronic final states with missing transverse energy using the variables $\alpha_{T}$ and b-quark multiplicity in pp collisions at $\sqrt{s}=8 \mathrm{TeV}$, Eur. Phys. J. C 73 (2013) 2568 [arXiv:1303.2985] [InSPIRE].

[28] S. Profumo and C.E. Yaguna, A statistical analysis of supersymmetric dark matter in the MSSM after WMAP, Phys. Rev. D 70 (2004) 095004 [hep-ph/0407036] [INSPIRE].

[29] N. Arkani-Hamed, A. Delgado and G.F. Giudice, The well-tempered neutralino, Nucl. Phys. B 741 (2006) 108 [hep-ph/0601041] [INSPIRE].

[30] L. Roszkowski, R. Ruiz de Austri, R. Trotta, Y.-L.S. Tsai and T.A. Varley, Global fits of the non-universal Higgs model, Phys. Rev. D 83 (2011) 015014 [arXiv:0903.1279] [INSPIRE].

[31] BayesFits Group collaboration, A. Fowlie, K. Kowalska, L. Roszkowski, E.M. Sessolo and Y.-L.S. Tsai, Dark matter and collider signatures of the MSSM, Phys. Rev. D 88 (2013) 055012 [arXiv:1306.1567] [INSPIRE].

[32] A. Kaminska, G.G. Ross and K. Schmidt-Hoberg, Non-universal gaugino masses and fine tuning implications for SUSY searches in the MSSM and the GNMSSM, JHEP 11 (2013) 209 [arXiv: 1308.4168] [INSPIRE].

[33] K. Kowalska, L. Roszkowski, E.M. Sessolo and S. Trojanowski, Low fine tuning in the MSSM with higgsino dark matter and unification constraints, JHEP 04 (2014) 166 [arXiv: 1402.1328] [INSPIRE].

[34] B.S. Acharya et al., Introducing the CTA concept, Astropart. Phys. 43 (2013) 3 [InSPIRE].

[35] CTA collaboration, M. Doro et al., Dark matter and fundamental physics with the Cherenkov Telescope Array, Astropart. Phys. 43 (2013) 189 [arXiv:1208.5356] [InSPIRE].

[36] M. Pierre, J.M. Siegal-Gaskins and P. Scott, Sensitivity of CTA to dark matter signals from the galactic center, JCAP 06 (2014) 024 [arXiv:1401.7330] [INSPIRE].

[37] B.C. Allanach, SOFTSUSY: a program for calculating supersymmetric spectra, Comput. Phys. Commun. 143 (2002) 305 [hep-ph/0104145] [INSPIRE].

[38] A. Djouadi, J.-L. Kneur and G. Moultaka, SuSpect: a fortran code for the supersymmetric and Higgs particle spectrum in the MSSM, Comput. Phys. Commun. 176 (2007) 426 [hep-ph/0211331] [INSPIRE].

[39] W. Porod, SPheno, a program for calculating supersymmetric spectra, SUSY particle decays and SUSY particle production at $e^{+} e^{-}$colliders, Comput. Phys. Commun. 153 (2003) 275 [hep-ph/0301101] [INSPIRE].

[40] S. Heinemeyer, W. Hollik and G. Weiglein, The masses of the neutral CP-even Higgs bosons in the MSSM: accurate analysis at the two loop level, Eur. Phys. J. C 9 (1999) 343 [hep-ph/9812472] [INSPIRE].

[41] S. Heinemeyer, W. Hollik and G. Weiglein, FeynHiggs: a program for the calculation of the masses of the neutral CP even Higgs bosons in the MSSM, Comput. Phys. Commun. 124 (2000) 76 [hep-ph/9812320] [INSPIRE].

[42] G. Degrassi, S. Heinemeyer, W. Hollik, P. Slavich and G. Weiglein, Towards high precision predictions for the MSSM Higgs sector, Eur. Phys. J. C 28 (2003) 133 [hep-ph/0212020] [INSPIRE]. 
[43] M. Frank et al., The Higgs boson masses and mixings of the complex MSSM in the Feynman-diagrammatic approach, JHEP 02 (2007) 047 [hep-ph/0611326] [INSPIRE].

[44] S.P. Martin, Three-loop corrections to the lightest Higgs scalar boson mass in supersymmetry, Phys. Rev. D 75 (2007) 055005 [hep-ph/0701051] [INSPIRE].

[45] R.V. Harlander, P. Kant, L. Mihaila and M. Steinhauser, Higgs boson mass in supersymmetry to three loops, Phys. Rev. Lett. 100 (2008) 191602 [Erratum ibid. 101 (2008) 039901] [arXiv:0803.0672] [INSPIRE].

[46] P. Kant, R.V. Harlander, L. Mihaila and M. Steinhauser, Light MSSM Higgs boson mass to three-loop accuracy, JHEP 08 (2010) 104 [arXiv: 1005.5709] [INSPIRE].

[47] T. Hahn, S. Heinemeyer, W. Hollik, H. Rzehak and G. Weiglein, High-precision predictions for the light CP-even Higgs boson mass of the MSSM, Phys. Rev. Lett. 112 (2014) 141801 [arXiv: 1312.4937] [INSPIRE].

[48] P. Draper, G. Lee and C.E.M. Wagner, Precise estimates of the Higgs mass in heavy SUSY, Phys. Rev. D 89 (2014) 055023 [arXiv:1312.5743] [InSPIRE].

[49] J.L. Feng, P. Kant, S. Profumo and D. Sanford, Three-loop corrections to the Higgs boson mass and implications for supersymmetry at the LHC, Phys. Rev. Lett. 111 (2013) 131802 [arXiv:1306.2318] [INSPIRE].

[50] O. Buchmueller et al., Implications of improved Higgs mass calculations for supersymmetric models, Eur. Phys. J. C 74 (2014) 2809 [arXiv:1312.5233] [INSPIRE].

[51] O. Buchmueller et al., The CMSSM and NUHM1 after LHC run 1, arXiv:1312.5250 [INSPIRE].

[52] LUX collaboration, D.S. Akerib et al., First results from the LUX dark matter experiment at the Sanford Underground Research Facility, Phys. Rev. Lett. 112 (2014) 091303 [arXiv: 1310.8214] [INSPIRE].

[53] A. Fowlie, A. Kalinowski, M. Kazana, L. Roszkowski and Y.L.S. Tsai, Bayesian implications of current LHC and XENON100 search limits for the constrained MSSM, Phys. Rev. D 85 (2012) 075012 [arXiv:1111.6098] [INSPIRE].

[54] L. Roszkowski, E.M. Sessolo and Y.-L.S. Tsai, Bayesian implications of current LHC supersymmetry and dark matter detection searches for the constrained MSSM, Phys. Rev. D 86 (2012) 095005 [arXiv: 1202.1503] [INSPIRE].

[55] P. Bechtle, S. Heinemeyer, O. Stål, T. Stefaniak and G. Weiglein, HiggsSignals: confronting arbitrary Higgs sectors with measurements at the Tevatron and the LHC, Eur. Phys. J. C 74 (2014) 2711 [arXiv: 1305.1933] [INSPIRE].

[56] P. Bechtle, O. Brein, S. Heinemeyer, G. Weiglein and K.E. Williams, HiggsBounds: confronting arbitrary Higgs sectors with exclusion bounds from LEP and the Tevatron, Comput. Phys. Commun. 181 (2010) 138 [arXiv:0811.4169] [INSPIRE].

[57] P. Bechtle, O. Brein, S. Heinemeyer, G. Weiglein and K.E. Williams, HiggsBounds 2.0.0: confronting neutral and charged Higgs sector predictions with exclusion bounds from LEP and the Tevatron, Comput. Phys. Commun. 182 (2011) 2605 [arXiv:1102.1898] [InSPIRE].

[58] P. Bechtle et al., HiggsBounds-4: improved tests of extended Higgs sectors against exclusion bounds from LEP, the Tevatron and the LHC, Eur. Phys. J. C 74 (2014) 2693 [arXiv:1311.0055] [INSPIRE]. 
[59] M. Drees, H. Dreiner, D. Schmeier, J. Tattersall and J.S. Kim, CheckMATE: confronting your favourite new physics model with LHC data, arXiv:1312.2591 [INSPIRE].

[60] A. Barr, C. Lester and P. Stephens, $m_{T 2}$ : the truth behind the glamour, J. Phys. G 29 (2003) 2343 [hep-ph/0304226] [INSPIRE].

[61] H.-C. Cheng and Z. Han, Minimal kinematic constraints and $m_{T 2}$, JHEP 12 (2008) 063 [arXiv:0810.5178] [INSPIRE].

[62] M. Cacciari and G.P. Salam, Dispelling the $N^{3}$ myth for the $k_{t}$ jet-finder, Phys. Lett. B 641 (2006) 57 [hep-ph/0512210] [INSPIRE].

[63] M. Cacciari, G.P. Salam and G. Soyez, The anti- $k_{t}$ jet clustering algorithm, JHEP 04 (2008) 063 [arXiv: 0802.1189] [InSPIRE].

[64] M. Cacciari, G.P. Salam and G. Soyez, FastJet user manual, Eur. Phys. J. C 72 (2012) 1896 [arXiv:1111.6097] [INSPIRE].

[65] DELPHES 3 collaboration, J. de Favereau et al., DELPHES 3, a modular framework for fast simulation of a generic collider experiment, JHEP 02 (2014) 057 [arXiv:1307.6346] [INSPIRE].

[66] C.G. Lester and D.J. Summers, Measuring masses of semiinvisibly decaying particles pair produced at hadron colliders, Phys. Lett. B 463 (1999) 99 [hep-ph/9906349] [INSPIRE].

[67] A.L. Read, Presentation of search results: the CL(s) technique, J. Phys. G 28 (2002) 2693 [INSPIRE].

[68] Particle Data Group collaboration, J. Beringer et al., Review of particle physics (RPP), Phys. Rev. D 86 (2012) 010001 [InSPIRE].

[69] K. Cheung, Y.-L.S. Tsai, P.-Y. Tseng, T.-C. Yuan and A. Zee, Global study of the simplest scalar phantom dark matter model, JCAP 10 (2012) 042 [arXiv: 1207.4930] [INSPIRE].

[70] XENON100 collaboration, E. Aprile et al., Dark matter results from 225 live days of XENON100 data, Phys. Rev. Lett. 109 (2012) 181301 [arXiv:1207.5988] [INSPIRE].

[71] G. Bélanger, F. Boudjema, A. Pukhov and A. Semenov, MicrOMEGAs3: a program for calculating dark matter observables, Comput. Phys. Commun. 185 (2014) 960 [arXiv: 1305.0237] [INSPIRE].

[72] J.R. Ellis, K.A. Olive and C. Savage, Hadronic uncertainties in the elastic scattering of supersymmetric dark matter, Phys. Rev. D 77 (2008) 065026 [arXiv:0801.3656] [InSPIRE].

[73] R.D. Young, Strange quark content of the nucleon and dark matter searches, PoS (LATTICE2012) 014 [arXiv: 1301.1765] [INSPIRE].

[74] J. Alwall, M. Herquet, F. Maltoni, O. Mattelaer and T. Stelzer, MadGraph 5: going beyond, JHEP 06 (2011) 128 [arXiv:1106.0522] [INSPIRE].

[75] T. Sjöstrand, S. Mrenna and P.Z. Skands, A brief introduction to PYTHIA 8.1, Comput. Phys. Commun. 178 (2008) 852 [arXiv:0710.3820] [INSPIRE].

[76] W. Beenakker, R. Hopker, M. Spira and P.M. Zerwas, Squark and gluino production at hadron colliders, Nucl. Phys. B 492 (1997) 51 [hep-ph/9610490] [INSPIRE].

[77] A. Kulesza and L. Motyka, Threshold resummation for squark-antisquark and gluino-pair production at the LHC, Phys. Rev. Lett. 102 (2009) 111802 [arXiv:0807.2405] [INSPIRE]. 
[78] A. Kulesza and L. Motyka, Soft gluon resummation for the production of gluino-gluino and squark-antisquark pairs at the LHC, Phys. Rev. D 80 (2009) 095004 [arXiv:0905.4749] [INSPIRE].

[79] W. Beenakker et al., Soft-gluon resummation for squark and gluino hadroproduction, JHEP 12 (2009) 041 [arXiv:0909.4418] [INSPIRE].

[80] W. Beenakker et al., Squark and gluino hadroproduction, Int. J. Mod. Phys. A 26 (2011) 2637 [arXiv: 1105.1110] [INSPIRE].

[81] ATLAS collaboration, Search for squarks and gluinos with the ATLAS detector in final states with jets and missing transverse momentum and $20.3 \mathrm{fb}^{-1}$ of $\sqrt{s}=8 \mathrm{TeV}$ proton-proton collision data, ATLAS-CONF-2013-047, CERN, Geneva Switzerland (2013).

[82] ATLAS collaboration, Search for strong production of supersymmetric particles in final states with missing transverse momentum and at least three b-jets using $20.1 \mathrm{fb}^{-1}$ of $p p$ collisions at $\sqrt{s}=8 \mathrm{TeV}$ with the ATLAS detector, ATLAS-CONF-2013-061, CERN, Geneva Switzerland (2013).

[83] K.N. Abazajian and J.P. Harding, Constraints on WIMP and Sommerfeld-enhanced dark matter annihilation from HESS observations of the galactic center, JCAP 01 (2012) 041 [arXiv:1110.6151] [INSPIRE].

[84] Fermi-LAT collaboration, M. Ackermann et al., Dark matter constraints from observations of 25 milky way satellite galaxies with the Fermi Large Area Telescope, Phys. Rev. D 89 (2014) 042001 [arXiv:1310.0828] [INSPIRE].

[85] K. Bernlöhr et al., Monte Carlo design studies for the Cherenkov Telescope Array, Astropart. Phys. 43 (2013) 171 [arXiv:1210.3503] [INSPIRE].

[86] BAYEsFITS Group collaboration, K. Kowalska et al., Constrained next-to-minimal supersymmetric Standard Model with a $126 \mathrm{GeV}$ Higgs boson: a global analysis, Phys. Rev. D 87 (2013) 115010 [arXiv:1211.1693] [INSPIRE].

[87] F. Feroz, M.P. Hobson and M. Bridges, MultiNest: an efficient and robust Bayesian inference tool for cosmology and particle physics, Mon. Not. Roy. Astron. Soc. 398 (2009) 1601 [arXiv: 0809.3437] [INSPIRE].

[88] F. Mahmoudi, SuperIso v2.3: a program for calculating flavor physics observables in supersymmetry, Comput. Phys. Commun. 180 (2009) 1579 [arXiv:0808.3144] [INSPIRE].

[89] C. Bobeth et al., $B_{s, d} \rightarrow \ell^{+} \ell^{-}$in the Standard Model with reduced theoretical uncertainty, Phys. Rev. Lett. 112 (2014) 101801 [arXiv:1311.0903] [INSPIRE].

[90] ATLAS, CDF, CMS and D0 collaborations, First combination of Tevatron and LHC measurements of the top-quark mass, arXiv:1403.4427 [INSPIRE].

[91] J.R. Ellis, T. Falk and K.A. Olive, Neutralino-stau coannihilation and the cosmological upper limit on the mass of the lightest supersymmetric particle, Phys. Lett. B 444 (1998) 367 [hep-ph/9810360] [INSPIRE].

[92] M. Drees and M.M. Nojiri, The neutralino relic density in minimal $N=1$ supergravity, Phys. Rev. D 47 (1993) 376 [hep-ph/9207234] [INSPIRE].

[93] K.L. Chan, U. Chattopadhyay and P. Nath, Naturalness, weak scale supersymmetry and the prospect for the observation of supersymmetry at the Tevatron and at the CERN LHC, Phys. Rev. D 58 (1998) 096004 [hep-ph/9710473] [INSPIRE]. 
[94] J.L. Feng, K.T. Matchev and T. Moroi, Multi-TeV scalars are natural in minimal supergravity, Phys. Rev. Lett. 84 (2000) 2322 [hep-ph/9908309] [INSPIRE].

[95] J.L. Feng, K.T. Matchev and T. Moroi, Focus points and naturalness in supersymmetry, Phys. Rev. D 61 (2000) 075005 [hep-ph/9909334] [INSPIRE].

[96] WMAP collaboration, E. Komatsu et al., Seven-year Wilkinson Microwave Anisotropy Probe (WMAP) observations: cosmological interpretation, Astrophys. J. Suppl. 192 (2011) 18 [arXiv: 1001.4538 ] [INSPIRE].

[97] ATLAS collaboration, Prospects for benchmark supersymmetry searches at the high luminosity LHC with the ATLAS detector, ATL-PHYS-PUB-2013-011, CERN, Geneva Switzerland (2013).

[98] T. Cohen et al., SUSY simplified models at 14, 33 and 100 TeV proton colliders, JHEP 04 (2014) 117 [arXiv: 1311.6480] [inSPIRE].

[99] A. Arbey, M. Battaglia and F. Mahmoudi, Supersymmetric heavy higgs bosons at the LHC, Phys. Rev. D 88 (2013) 015007 [arXiv: 1303.7450] [INSPIRE].

[100] CMS collaboration, Search for MSSM neutral Higgs bosons decaying to tau pairs in pp collisions, CMS-PAS-HIG-12-050, CERN, Geneva Switzerland (2012).

[101] M.S. Carena, S. Heinemeyer, C.E.M. Wagner and G. Weiglein, Suggestions for benchmark scenarios for MSSM Higgs boson searches at hadron colliders, Eur. Phys. J. C 26 (2003) 601 [hep-ph/0202167] [INSPIRE].

[102] A. Djouadi and J. Quevillon, The MSSM Higgs sector at a high $M_{S U S Y}$ : reopening the low $\tan \beta$ regime and heavy Higgs searches, JHEP 10 (2013) 028 [arXiv:1304.1787] [INSPIRE].

[103] XENON1T collaboration, E. Aprile, The XENON1T dark matter search experiment, arXiv:1206.6288 [INSPIRE].

[104] M. Wood et al., Prospects for indirect detection of dark matter with CTA, arXiv: 1305.0302 [INSPIRE].

[105] J.F. Navarro, C.S. Frenk and S.D.M. White, The structure of cold dark matter halos, Astrophys. J. 462 (1996) 563 [astro-ph/9508025] [INSPIRE].

[106] J. Einasto, On the construction of a composite model for the galaxy and on the determination of the system of galactic parameters, Trudy Astrofiz. Inst. Alma-Ata 5 (1965) 87.

[107] B. Cabrera, L.M. Krauss and F. Wilczek, Bolometric detection of neutrinos, Phys. Rev. Lett. 55 (1985) 25 [INSPIRE].

[108] J. Monroe and P. Fisher, Neutrino backgrounds to dark matter searches, Phys. Rev. D 76 (2007) 033007 [arXiv:0706.3019] [INSPIRE].

[109] J. Billard, L. Strigari and E. Figueroa-Feliciano, Implication of neutrino backgrounds on the reach of next generation dark matter direct detection experiments, Phys. Rev. D 89 (2014) 023524 [arXiv: 1307.5458] [INSPIRE]. 